Review

\title{
Safety of Immunosuppressive Drugs Used as Maintenance Therapy in Kidney Transplantation: A Systematic Review and Meta-Analysis
}

Celline Cardoso Almeida ${ }^{1}{ }^{*}$, Micheline Rosa Silveira ${ }^{2}$, Vânia Eloisa de Araújo ${ }^{2}$, Livia Lovato Pires de Lemos ${ }^{2}$, Juliana de Oliveira Costa ${ }^{2}$, Carlos Augusto Lins Reis ${ }^{2}$, Francisco de Assis Acurcio ${ }^{2}$ and Maria das Gracas Braga Ceccato ${ }^{2}$

1 Faculdade de Medicina, Universidade Federal de Minas Gerais, Av. Prof. Alfredo Balena, 190, Belo Horizonte/MG, Brazil

2 Faculdade de Farmácia, Universidade Federal de Minas Gerais, Av. Antônio Carlos, 6627, Campus Pampulha, Belo Horizonte/MG, Brazil; E-Mails: michelinerosa@gmail.com (M.R.S.); vaniaearaujo@gmail.com (V.E.A.); lilolemos@gmail.com (L.L.P.L.); juliana.olic@gmail.com (J.O.C.); carloslinsr@gmail.com (C.A.L.R.); fracurcio@gmail.com (F.A.A.); mgbceccato@gmail.com (M.G.B.C.)

* Author to whom correspondence should be addressed; E-Mail: celline.cardoso@gmail.com; Tel.: +1-55-31-8861-9577.

Received: 9 July 2013; in revised form: 3 September 2013 / Accepted: 16 September 2013 / Published: 30 September 2013

Abstract: To evaluate the safety of regimens containing calcineurin inhibitors (CNI), proliferation signal inhibitors (TOR-I) and antimetabolites, we conducted a meta-analysis of randomized clinical trials (RCTs) and observational studies. A total of 4,960 citations were identified in our electronic search and 14 additional articles were identified through hand searching. Forty-eight articles (11,432 participants) from 42 studies (38 RCTs and four cohorts) met the inclusion criteria. Meta-analysis results revealed the following: (i) tacrolimus was associated with an increased risk for diabetes and lower risk of dyslipidemia, compared to cyclosporine; (ii) mycophenolate mofetil (MMF) was associated with increased risk for total infections, abdominal pain, diarrhea and vomiting, compared with azathioprine; (iii) sirolimus was associated with higher risk of anemia, diabetes, dyslipidemia, lymphoceles and withdrawal compared to tacrolimus or cyclosporine, and cyclosporine was associated with an increased risk of CMV infection; (iv) the combination of CNI with antimetabolites was associated with more adverse events than CNI alone; (v) TOR-I was related to more adverse events than MMF. The data observed 
in this meta-analysis are similar to those describe by others authors; thus, the choice of treatment must be made by the clinical staff based on specific patient characteristics.

Keywords: kidney transplantation; immunosuppressive drugs; adverse events; meta-analysis

\section{Introduction}

Chronic kidney disease (CKD), most commonly defined as persistent impaired kidney function [1], is a comorbid condition with multiple manifestations that is recognized as an important worldwide public health problem. The prevalence of CKD has increased over the years. It is strongly related with ageing and is more prevalent among women [2-5] and African Americans [6].

A systematic review of population based studies [7] confirmed that impaired kidney function (iKF) is as common as diabetes mellitus in the general population, and higher quality studies have reported the prevalence of $\mathrm{iKF}$ to range from $1.7 \%$ in China to $8.1 \%$ in the United States. To reduce disease progression, there have been increasing efforts to promote early diagnosis of CKD [8,9]. Without proper care, CKD leads to complications of reduced kidney function, increased risk of cardiovascular disease and, ultimately, kidney failure, the need of renal replacement therapy (RRT) (namely the end-stage renal disease) and death [1].

In terms of RRT, renal transplantation (RT) reduces disability, and improves kidney function and quality of life, and it also provides greater life expectancy and is more cost-effective compared with dialysis [10-12]. In 2008, 547,982 United States residents were treated for end-stage renal disease and 17,413 transplants were performed [13].

The effectiveness of immunosuppressive drugs for maintenance therapy has not been a direct issue regarding the success of RT. Nevertheless, choosing the best suitable immunosuppressive therapy is still fairly complex. Multiple classes of drugs are used in combination. Usually, steroids are administrated with calcineurin inhibitors (CNI) such as cyclosporine (CsA) or tacrolimus (TAC) and either proliferation signal inhibitors (TOR-I) such as sirolimus (SRL) and everolimus (EVL) or antimetabolites (AMETAB) such as azathioprine (AZA) or mycophenolate mofetil (MMF) [14].

Moreover, the balance of advantages and disadvantages is used to determine the regimen of choice, because an increasing range of adverse events must be considered when deciding on the optimal immunosuppressive strategy for an individual patient.

New-onset diabetes mellitus (NODAT) is highly associated with CNI treatment, whereas CMV infection is associated with antimetabolites, and dyslipidemia is associated with TOR-I $[14,15]$. Thus, it is critical to identify and quantify which adverse events are related to a certain drug regimen as this information is missing in the literature.

Therefore, this systematic review and meta-analysis of randomized controlled trials and observational studies was conducted to evaluate the safety of the most commonly used immunosuppressive regimens. The availability of such information would be useful for clinicians when deciding which treatment is most appropriate for each patient. 


\section{Literature Search}

This systematic review is reported in accordance with Preferred Reporting Items for Systematic Reviews and Meta-analysis (PRISMA) [16].

\subsection{Eligibility Criteria and Study Selection}

Randomized Controlled Trials (RCTs) and cohort studies comparing treatment regimens that included the immunosuppressants azathioprine, cyclosporine, tacrolimus, mycophenolate mofetil or enteric mycophenolate, sirolimus, or everolimus in any dose and with at least 6 months follow-up were included in this analysis. The eligibility criteria for participants included end-stage renal disease patients over age 16 who had undergone renal transplantation for the first time or not, with a living or deceased donor. Only studies published in English, Portuguese or Spanish were included.

Studies with the following characteristics were excluded: (i) enrolled patients younger than 16 years of age; (ii) did not evaluate RT exclusively; (iii) presented pharmacokinetics or pharmacodynamics results; (iv) were single-arm studies; (v) were non-randomized controlled trials; (vi) were placebo controlled studies; (vii) described results from less than 6 months follow-up; (viii) assessed induction therapy; (ix) were health technology assessments studies; and (x) were studies with a conversion of drugs.

The present study focused on the safety of immunosuppressive drugs; thus, studies that did not report safety information were excluded.

\subsection{Search Strategy}

Several article searches were performed in the Pubmed/MEDLINE, Cochrane Controlled Trials Register, Cochrane's Renal Group and LILACS databases, covering the period from the inception of the database until August 2013. We also performed a manual search of references that were included in the identified studies and systematic reviews $[17,18]$.

Various combinations of terms were used to search the electronic databases, including terms referring to the disease, interventions and the type of study: "immunosuppression", "transplant", "kidney", "renal”, "azathioprine", "mycophenolate mofetil”, “cyclosporine", "tacrolimus", "sirolimus", “everolimus", "effectiveness", "efficacy" and "safety".

\subsection{Selection of Studies and Data Collection}

Two independent reviewers performed the study selection in three phases: analyses of titles, abstracts and full-text articles. A third reviewer resolved disagreements regarding eligibility.

After meticulous reading of all included articles, data were extracted in especially designed manual and electronic forms, using Cochrane Review Manager Software-Revman ${ }^{\circledR} 5.1$ (The Nordic Cochrane Center, Købehvn, Denmark). The studies were classified according to its treatment strategyarms. Comparison between treatment arms was possible if the schemes contained the same concomitant medication (e.g., steroids) at the same dose, differing only by the drug used as intervention and the control.

Any drug-related adverse event and withdrawals were considered safety outcomes. The overall safety outcomes were collected to extract those most prevalent among them. The data were collected in 
terms of the number of patients who presented a specific outcome. All consolidated data were reviewed to avoid typing errors.

\subsection{Quality Assessment}

The quality of the study was independently accessed by two reviewers, and any disagreements were resolved by consensus. Randomized controlled trials were evaluated using the Cochrane Collaboration Tool [19] considering the following items: random sequence generation, allocation concealment, blinding, and incomplete outcome data. Open label studies were not considered risk of bias. Observational studies were evaluated using Newcastle scale [20].

\subsection{Data Synthesis and Data Analysis}

Outcomes were meta-analyzed if they were reported in at least two articles, within the same treatment arm and at the same time of follow-up. Random effect models were employed to estimate the pooled effect sizes across studies [21]. The results are expressed as the relative risk (RR) with $95 \%$ confidence interval $(95 \% \mathrm{CI}) ; p$ value $<0.05$ was considered significant. To assess heterogeneity $\mathrm{I}^{2}$ and $p$ values were used $\left(\mathrm{I}^{2}>50 \%\right.$ and $p<0.05$ indicated high heterogeneity) Publication bias was accessed using funnel plot. Single analysis (estimated RR from raw data) was performed if the data was not eligible to enter in meta-analysis. All analysis was conducted using Revman 5.1.

\section{Results}

\subsection{Study Characteristics}

A PRISMA flow chart describing the publication screening process and the reasons for exclusion is shown in Figure 1A total of 5,875 citations identified by the electronic search, and 16 additional articles were identified via manual searching. A total of 48 articles (11,432 participants), from 42 studies, 38 RCTs [22-65] and four cohorts [66-69] met the inclusion criteria.

Table 1 shows the characteristics of included studies organized by treatment strategy, according to the treatment protocol of each study. CsA was the most prevalent drug in all schemes, as it was used in 34 articles. The majority of studies (33\%) compared TAC versus (vs.) CsA, usually using an antimetabolite and a steroid in combination. Within each study, differences between groups in terms of gender, race, age and allograft characteristics were not significant, indicating that the allocation of participants into the treatment groups was satisfactory. Twenty five RCTs were multicenter studies, with the number of centers ranging from 2 to 65 centers. Nearly $50 \%$ of the studies were conducted in European countries, $21 \%$ of the studies were conducted in the United States, and $14 \%$ were conducted in Brazil. 
Figure 1. Flow chart of studies included in the systematic review.

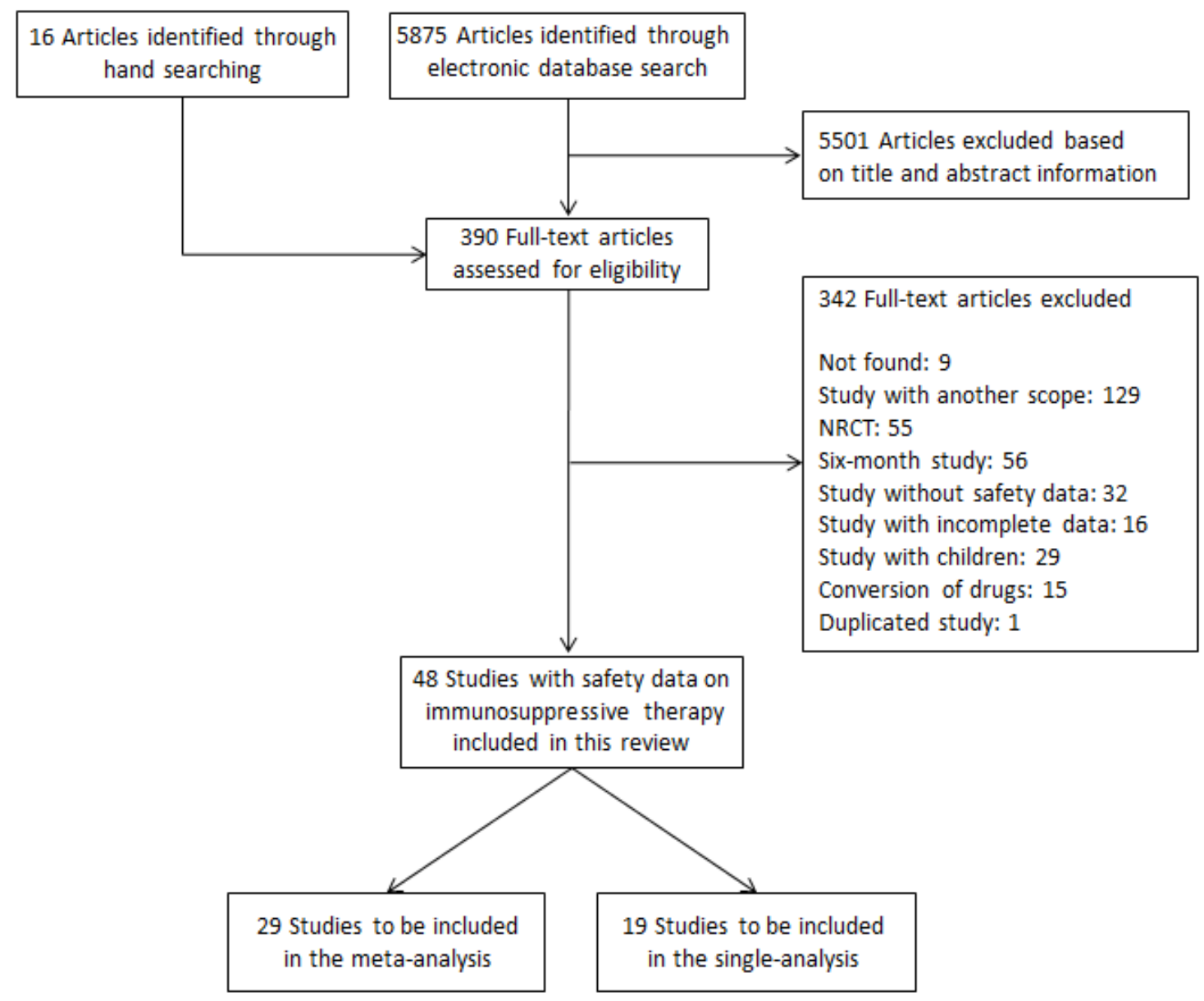

Of the 38 RCT studies evaluating immunosuppressants only 13 (34\%) reported adequate sequence generation and most studies did not report the allocation concealment (73\%). One study [34] used the numbers of records for randomization and was classified as high risk for selection bias. All studies, but four $[22,23,26,40]$ used intention to treat or modified intention to treat analysis. A summary of RCT quality is shown in Figure 2. The four included cohorts [66-69] assigned three or four stars in the selection domain, one star in the comparability of groups and one to two in the exposure, demonstrating good quality. Funnel plots of meta-analyses were all symmetrical, indicating the absence of bias.

Figure 2. Quality of RCTs included in the review.

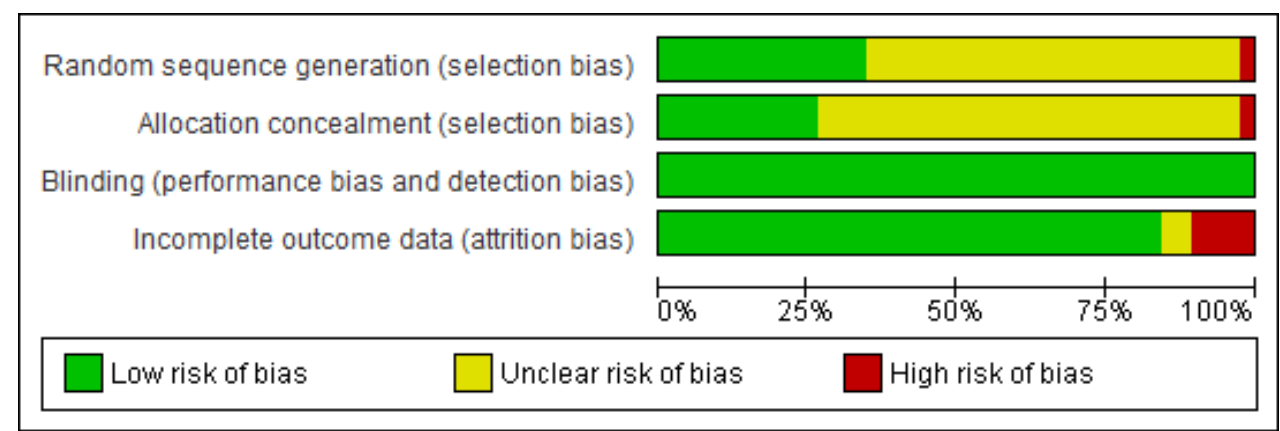


Table 1. Characteristics of included studies.

\begin{tabular}{|c|c|c|c|c|c|c|c|c|c|}
\hline Study (year) & Treatment & $\begin{array}{c}\text { N (female } \\
\%)\end{array}$ & $\begin{array}{c}\text { White } \\
\%\end{array}$ & $\begin{array}{c}\text { First } \\
\text { Transplant \% } \\
\end{array}$ & $\begin{array}{l}\text { Deceased } \\
\text { Donor \% }\end{array}$ & $\begin{array}{c}\text { Cold Ischemia time } \\
\text { (SD) }\end{array}$ & $\begin{array}{l}\text { Mean Donor Age } \\
\text { (SD) }\end{array}$ & $\begin{array}{l}\text { Mean Age } \\
\text { (SD) }\end{array}$ & $\begin{array}{l}\text { Study design, location, time } \\
\text { of follow-up and funding }\end{array}$ \\
\hline \multicolumn{10}{|c|}{ 1. CNI vs. CNI } \\
\hline 1.Scantleburry & CsA + Pred & 20 & NR & 100 & NR & NR & NR & NR & \multirow{4}{*}{$\begin{array}{l}\text { RCT, USA, } \\
\text { single center, } 12 \text { months } \\
\text { RCT, England, multicenter (15), } \\
\text { open label, } 12 \text { months, funded by } \\
\text { Fujisawa GMBH }\end{array}$} \\
\hline (1991) [22] & $\mathrm{TAC}+$ Pred & 14 & NR & 100 & NR & NR & NR & NR & \\
\hline 2. Mayer & $\mathrm{CsA}+\mathrm{AZA}+$ Pred & $145(36.6)$ & NR & 89.7 & 100 & NR & 43.0 & 45.8 & \\
\hline (1997) [23] & $\mathrm{TAC}+\mathrm{AZA}+$ Pred & $303(35.3)$ & NR & 90.4 & 100 & NR & 45.2 & 46.6 & \\
\hline 3. Yang & $\mathrm{CsA}+\mathrm{MMF}+$ Ster & $30(37)$ & 87.0 & NR & 57.0 & $15(1.6)$ & $37(2.6)$ & $48.0(2.2)$ & \multirow{2}{*}{$\begin{array}{l}\text { RCT, USA, single center, } \\
\text { open label, } 12 \text { months }\end{array}$} \\
\hline (1999) [24] & $\mathrm{TAC}+\mathrm{MMF}+$ Ster & $30(50)$ & 77.0 & NR & 67.0 & $14(1.5)$ & $39(3.0)$ & $45.0(2.4)$ & \\
\hline 4. Wang & $\mathrm{CsA}+\mathrm{MMF}+$ Pred & 32 & NR & NR & 100 & NR & NR & $281,(187)$ & \multirow{2}{*}{$\begin{array}{l}\text { RCT, China, single center, } \\
12 \text { months results }\end{array}$} \\
\hline (2000) [25] & $\mathrm{TAC}+\mathrm{MMF}+$ Pred & 25 & NR & NR & 100 & NR & NR & $38.1(18 . /)$ & \\
\hline 5. Nichelle & $\mathrm{CsA}+\mathrm{AZA}+\mathrm{Ster}$ & 46 & NR & NR & NR & NR & NR & NR & \multirow{4}{*}{$\begin{array}{l}\text { RCT, France, single center, } \\
12 \text { and } 36 \text { months } \\
\text { RCT, Brazil, multicenter (15), } \\
\text { open label, } 12 \text { months }\end{array}$} \\
\hline (2002) [26] & $\mathrm{TAC}+\mathrm{AZA}+$ Ster & 48 & NR & NR & NR & NR & NR & NR & \\
\hline 6. Campos & CsA & $81(44)$ & NR & 94 & 52.0 & NR & $37.5(14.3)$ & $40.9(12.3)$ & \\
\hline (2002) [27] & $\mathrm{TAC}$ & $85(52)$ & NR & 96 & 46.0 & NR & $36.5(13.7)$ & $40.5(10.7)$ & \\
\hline $\begin{array}{l}\text { 7. Murphy } \\
\text { (2003) [28] }\end{array}$ & $\begin{array}{l}\text { CsA }+ \text { Pred }+ \text { AZA } \\
\text { TAC }+ \text { Pred }+ \text { AZA }\end{array}$ & $\begin{array}{l}50(30.0) \\
52(38.5)\end{array}$ & $\begin{array}{l}\text { NR } \\
\text { NR }\end{array}$ & $\begin{array}{l}88.0 \\
88.0\end{array}$ & $\begin{array}{l}84.0 \\
82.0\end{array}$ & $\begin{array}{l}\text { LD:1.7; CAD:19.0; } \\
\text { NHBD:15.5; LD: } 2.2 ; \\
\text { CAD:18.7; NHBD:15.1 }\end{array}$ & $\begin{array}{c}\text { LD:49; CAD:44; } \\
\text { NHBD:48; LD:45; } \\
\text { CAD:39; NHBD:49 }\end{array}$ & $\begin{array}{l}45.0(12.0) \\
45.0(14.0)\end{array}$ & $\begin{array}{l}\text { RCT, England, multicenter (2), } \\
\text { open label, } 12 \text { months }\end{array}$ \\
\hline 8. Jurewicz & $\mathrm{CsA}+\mathrm{AZA}+$ Ster & 117 & NR & NR & NR & NR & NR & NR & \multirow{2}{*}{$\begin{array}{l}\text { Cohort, United Kingdom, } \\
\text { single center, } 72 \text { months }\end{array}$} \\
\hline (2003) [66] & $\mathrm{TAC}+\mathrm{AZA}+\mathrm{Ster}$ & 115 & NR & NR & NR & NR & NR & NR & \\
\hline 9. Hardinger & $\mathrm{CsA}+\mathrm{AZA}+$ Ster & $66(39)$ & 79 & 100 & 51.0 & $12(4)$ & NR & $44,0(13.0)$ & \multirow{2}{*}{$\begin{array}{l}\text { RCT, USA, single center, } \\
\text { open label, } 12 \text { months }\end{array}$} \\
\hline (2005) [29] & $\mathrm{TAC}+\mathrm{AZA}+\mathrm{Ster}$ & $134(36)$ & 79 & 100 & 58.0 & $13(5)$ & NR & $46,0(13.0)$ & \\
\hline 10. Fukuhara & CsA + Pred & $137(36.5)$ & NR & NR & 100 & $11.95(6.12)$ & $47(18)$ & $44(9)$ & \multirow{2}{*}{$\begin{array}{l}\text { Cohort, Japan, } \\
\text { single Center, } 10 \text { years }\end{array}$} \\
\hline (2005) [67] & $\mathrm{TAC}+$ Pred & $55(30.9)$ & NR & NR & 100 & $12.13(6.58)$ & $46(16)$ & $42(11)$ & \\
\hline 11. Silva & $\mathrm{CsA}+\mathrm{AZA} / \mathrm{MMF}$ & $80(44)$ & 50 & 91 & 100 & $21(8)$ & $34(14.0)$ & $42(12.0)$ & \multirow{2}{*}{$\begin{array}{l}\text { Cohort, Brazil, } \\
\text { single center, } 12 \text { months }\end{array}$} \\
\hline (2006) [68] & $\mathrm{TAC}+\mathrm{AZA} / \mathrm{MMF}$ & $68(50)$ & 53 & 85 & 100 & $18(7)$ & $34(12.0)$ & $43(12.0)$ & \\
\hline \multirow{3}{*}{$\begin{array}{l}\text { 12. Silva, Jr. } \\
(2007)[30]\end{array}$} & CsA + Pred & $212(35.5)$ & 76.9 & 95.8 & 47.6 & $18.44(7.11)$ & NR & $47.6(13.0)$ & \multirow{3}{*}{$\begin{array}{l}\text { RCT, Brazil, , multicenter ( } 60) \text {, } \\
\text { open label, } 12 \text { months, funded } \\
\text { by AstellasPharma US }\end{array}$} \\
\hline & TAC + Pred & $212(35.8)$ & 71.7 & 96.3 & 50.0 & $19.41(7.27)$ & NR & $48.6(12.9)$ & \\
\hline & TAC XL + Pred & $214(38.7)$ & 74.8 & 96.3 & 51.9 & $17.88(7.73)$ & NR & $47.8(13.0)$ & \\
\hline 13. Cheung & CsA + Pred & $38(34.2)$ & $100^{\mathrm{a}}$ & 100 & 100 & $8.7(4.6)$ & $48.9(13.2)$ & $40.2(11.7)$ & \multirow{4}{*}{$\begin{array}{l}\text { RCT, China, single center, } \\
\text { open label, } 60 \text { months } \\
\text { RCT, USA, multicenter (5), } \\
\text { open label, } 12 \text { months }\end{array}$} \\
\hline (2009) [31] & $\mathrm{TAC}+$ Pred & $38(44.8)$ & $100^{\mathrm{a}}$ & 100 & 100 & $9.1(5.1)$ & $48.9(13.2)$ & $41.8(7.5)$ & \\
\hline 14. Vicenti & CsA & $28(21.4)$ & 53.6 & 100 & NR & NR & NR & 46.6 & \\
\hline (1996) [32] & TAC & $92(34.8)$ & 51.1 & 100 & NR & NR & NR & 44.1 & \\
\hline
\end{tabular}


Table 1. Cont

\begin{tabular}{|c|c|c|c|c|c|c|c|c|c|}
\hline Study (year) & Treatment & $\begin{array}{c}\text { N (female } \\
\%)\end{array}$ & $\begin{array}{c}\text { White } \\
\%\end{array}$ & $\begin{array}{c}\text { First } \\
\text { Transplant \% } \\
\end{array}$ & $\begin{array}{l}\text { Deceased } \\
\text { Donor \% }\end{array}$ & $\begin{array}{l}\text { Cold Ischemia time } \\
\text { (SD) }\end{array}$ & $\begin{array}{l}\text { Mean Donor Age } \\
\text { (SD) }\end{array}$ & $\begin{array}{l}\text { Mean Age } \\
\text { (SD) }\end{array}$ & $\begin{array}{l}\text { Study design, location, time } \\
\text { of follow-up and funding }\end{array}$ \\
\hline \multicolumn{10}{|c|}{ 2. CNI vs. CNI + AMETAB } \\
\hline \multirow{5}{*}{$\begin{array}{l}\text { 1. Moreso } \\
\text { (1998) [33] }\end{array}$} & CsA + Pred & & & & & & & & \multirow{5}{*}{$\begin{array}{l}\text { RCT, Spain, multicenter (2), } \\
\text { double blind in the standard } \\
\text { dose CsA groups and open label } \\
\text { in the low-dose CsA, } 24 \text { months }\end{array}$} \\
\hline & CsA + MMF(ld $)+$ & $27(48.2)$ & NR & 37.0 & 100 & NR & $41(16)$ & $47(15)$ & \\
\hline & Pred & $27(44.4)$ & NR & 44.4 & 100 & NR & $41(18)$ & $45(14)$ & \\
\hline & $\mathrm{CsA}+\mathrm{MMF}+$ Pred & $28(42.9)$ & NR & 46.4 & 100 & NR & $42(17)$ & $43(15)$ & \\
\hline & $\begin{array}{c}\mathrm{CsA}(\mathrm{ld})+\mathrm{MMF}+ \\
\text { Pred }\end{array}$ & $15(33.3)$ & NR & 93.4 & 100 & NR & $44(14)$ & $47(7)$ & \\
\hline 2. Raofi & $\mathrm{CsA}+\mathrm{AZA}$ & $21(27.7)$ & $100^{\mathrm{b}}$ & 100 & 100 & $26(10)$ & NR & $46.0(11.0)$ & \multirow{5}{*}{$\begin{array}{l}\text { RCT, USA, } \\
\text { single center, } 12 \text { months } \\
\text { RCT, Italy, } \\
\text { single center, } 60 \text { months } \\
\text { RCT, Italy and Spain, } \\
\text { multicenter (36), } \\
\text { open label, } 12 \text { and } 36 \text { months }\end{array}$} \\
\hline (1999) [34] & $\mathrm{TAC}+$ Pred & $14(22.9)$ & $100^{\mathrm{b}}$ & 100 & 100 & $25(8)$ & NR & $44.0(14.0)$ & \\
\hline 3. Sandrini (2000) & CsA + Pred & $58(45.0)$ & NR & 100 & 100 & NR & $35(14)$ & $42(11)$ & \\
\hline$[35]$ & $\mathrm{CsA}+\mathrm{AZA}+$ Pred & $58(38.0)$ & NR & 100 & 100 & NR & $35(16)$ & $44(10)$ & \\
\hline $\begin{array}{l}\text { 4. Segoloni } \\
(2000)[36] \\
{[\text { Pascual }(2003)][37]}\end{array}$ & $\begin{array}{c}\mathrm{TAC}+\text { Pred } \\
\mathrm{TAC}+\mathrm{AZA}+\text { Pred }\end{array}$ & $\begin{array}{l}236(35.2) \\
239(35.6)\end{array}$ & $\begin{array}{l}\text { NR } \\
\text { NR }\end{array}$ & $\begin{array}{l}\text { NR } \\
\text { NR }\end{array}$ & $\begin{array}{l}100 \\
100\end{array}$ & $\begin{array}{l}18.0 \\
17.6\end{array}$ & $\begin{array}{l}\mathrm{NR} \\
\mathrm{NR}\end{array}$ & $\begin{array}{l}46.0 \\
45.0\end{array}$ & \\
\hline $\begin{array}{l}\text { 5. Chang } \\
(2001)[38]\end{array}$ & $\begin{array}{c}\mathrm{TAC}+\text { Ster } \\
\mathrm{TAC}+\mathrm{AZA}+\text { Ster }\end{array}$ & $\begin{array}{l}124(37.9) \\
121(32.2)\end{array}$ & $\begin{array}{l}77,4 \\
76,0\end{array}$ & $\begin{array}{l}\text { NR } \\
\text { NR }\end{array}$ & $\begin{array}{l}\text { NR } \\
\text { NR }\end{array}$ & $\begin{array}{l}20.4 \\
21.3\end{array}$ & $\begin{array}{l}\text { NR } \\
\text { NR }\end{array}$ & $\begin{array}{l}48.0 \\
45.0\end{array}$ & $\begin{array}{l}\text { RCT, United Kingdom, } \\
\text { multicenter (08), } \\
\text { open label, } 12 \text { months }\end{array}$ \\
\hline $\begin{array}{l}\text { 6. Squiflet } \\
\text { (2001) [39] }\end{array}$ & $\begin{array}{c}\text { TAC }+ \text { Pred } \\
\text { TAC }+ \text { MMF }(\mathrm{ld})+ \\
\text { Pred } \\
\text { TAC }+ \text { MMF }+ \text { Pred }\end{array}$ & $\begin{array}{l}82(46.3) \\
79(32.9) \\
71(36.6)\end{array}$ & $\begin{array}{l}93.9 \\
97.5 \\
95.8\end{array}$ & $\begin{array}{l}86.6 \\
87.3 \\
90.1\end{array}$ & $\begin{array}{l}100 \\
100 \\
100\end{array}$ & $\begin{array}{l}\text { NR } \\
\text { NR } \\
\text { NR }\end{array}$ & $\begin{array}{l}45.6(18.1) \\
45.6(16.0) \\
45.4(16.9)\end{array}$ & $\begin{array}{l}46.6(14.5) \\
46.5(13.3) \\
48.0(13.3)\end{array}$ & $\begin{array}{l}\text { RCT, Belgium, multicenter (16), } \\
12 \text { months, funded by Fujisawa }\end{array}$ \\
\hline \multicolumn{10}{|c|}{ 3. CNI vs. AMETAB } \\
\hline 1. Hall & CsA & $138(42.8)$ & NR & 100 & 100 & 22.0 & NR & NR & RCT, Australia, multicenter (7), \\
\hline (1988) [40] & $\mathrm{AZA}+$ Pred & $138(45.0)$ & NR & 100 & 100 & 22.7 & NR & NR & 36 months, funded by Sandoz \\
\hline 2. Schnuelle & $\mathrm{CsA}+$ Ster & $44(27.3)$ & NR & 95,5 & NR & $21.7(9.0)$ & $40.7(15.3)$ & $44.7(13.3)$ & RCT, Germany, multicenter (3), \\
\hline (2001) [41] & MMF + Ster & $40(45.0)$ & NR & 97,5 & NR & $21.0(7.5)$ & $47.7(15.4)$ & $51.3(11.5)$ & open label,12 months \\
\hline 3. Hamdy & $\mathrm{TAC}+\mathrm{SRL}+$ Pred & $65(20.0)$ & NR & 100 & 0 & NR & $35.6(10.3)$ & $32.3(10.3)$ & RCT, Egypt, \\
\hline$(2008)[42]$ & $\mathrm{MMF}+\mathrm{SRL}+$ Pred & $67(29.8)$ & NR & 100 & 0 & NR & $36.2(10.2)$ & $31.8(8.6)$ & single center, 63 months \\
\hline
\end{tabular}


Table 1. Cont.

\begin{tabular}{|c|c|c|c|c|c|c|c|c|c|}
\hline Study (year) & Treatment & $\begin{array}{c}\text { N (female } \\
\%)\end{array}$ & $\begin{array}{l}\text { White } \\
\%\end{array}$ & $\begin{array}{c}\text { First } \\
\text { Transplant \% }\end{array}$ & $\begin{array}{l}\text { Deceased } \\
\text { Donor \% }\end{array}$ & $\begin{array}{l}\text { Cold Ischemia time } \\
\text { (SD) }\end{array}$ & $\begin{array}{l}\text { Mean Donor Age } \\
\text { (SD) }\end{array}$ & $\begin{array}{l}\text { Mean Age } \\
\text { (SD) }\end{array}$ & $\begin{array}{l}\text { Study design, location, time } \\
\text { of follow-up and funding }\end{array}$ \\
\hline \multicolumn{10}{|c|}{ 4. CNI vs. TOR-I } \\
\hline \multirow{2}{*}{$\begin{array}{l}\text { 1. Groth } \\
(1998)[43]\end{array}$} & $\mathrm{CsA}+\mathrm{AZA}+$ Pred & $42(40.0)$ & 88.0 & 100 & 100 & $17.4(7.2)$ & $37.7(15.9)$ & $41.6(11.8)$ & \multirow{2}{*}{$\begin{array}{l}\text { RCT, Sweden, multicenter (11), } \\
\text { open label, } 12 \text { months }\end{array}$} \\
\hline & $\mathrm{SRL}+\mathrm{AZA}+$ Pred & $41(29.0)$ & 98.0 & 100 & 100 & $18.9(7.4)$ & $44.6(13.4)$ & $47.5(10.8)$ & \\
\hline \multicolumn{10}{|l|}{ 2. Büchler } \\
\hline$(2007)[44]$ & $\mathrm{CsA}+\mathrm{MMF}+$ Ster & $74(39.2)$ & 95.9 & 89.2 & 100 & $20.17(5.46)$ & $41.3(14.0)$ & $45.1(12.4)$ & \multirow{2}{*}{$\begin{array}{l}\text { RCT, France, multicenter (13), } \\
12 \text { months, funded by Wyeth }\end{array}$} \\
\hline $\begin{array}{l}\text { Lebranchu } \\
\text { (2012) [45] }\end{array}$ & $\mathrm{SRL}+\mathrm{MMF}+\mathrm{Ster}$ & $71(38.0)$ & 94.4 & 95.8 & 100 & $19.30(5.24)$ & $38.7(14.4)$ & $45.6(10.3)$ & \\
\hline \multirow{2}{*}{$\begin{array}{l}\text { 3. Guba } \\
(2010)[46]\end{array}$} & $\mathrm{CsA}+\mathrm{MMF}+\mathrm{Ster}$ & 71 & 98.6 & 89.9 & 88.4 & $13.0(7.0)$ & $47.1(14.3)$ & $47.1(11.1)$ & \multirow{2}{*}{$\begin{array}{l}\text { RCT, Germany, multicenter (9), } \\
12 \text { months, funded by Wyeth } \\
\text { and Fresenius Biotech }\end{array}$} \\
\hline & $\mathrm{SRL}+\mathrm{MMF}+\mathrm{Ster}$ & 70 & 98.6 & 94.4 & 90.1 & $12.1(5.7)$ & $46.9(14.3)$ & $47.0(10.8)$ & \\
\hline \multirow{2}{*}{$\begin{array}{l}\text { 4.Glotz } \\
(2010)[47]\end{array}$} & $\mathrm{TAC}+\mathrm{MMF}+$ Ster & 70 & 91.4 & 94.3 & 100 & $18(6)$ & $45.1(12.6)$ & $46.7(10.6)$ & \multirow{2}{*}{$\begin{array}{l}\text { RCT, France, multicenter (13), } \\
12 \text { months, funded by Wyeth }\end{array}$} \\
\hline & $\mathrm{SRL}+\mathrm{MMF}+$ Ster & 71 & 77.5 & 94.4 & 100 & $19(5)$ & $45.2(13.4)$ & $48.5(9.5)$ & \\
\hline \multicolumn{10}{|c|}{ 5. CNI + AMETAB vs. CNI + AMETAB vs. CNI + AMETAB } \\
\hline \multirow{3}{*}{$\begin{array}{l}\text { 1. Hernandez } \\
\text { (2007) [48] }\end{array}$} & $\mathrm{CsA}+\mathrm{AZA}+\mathrm{Ster}$ & $80(26.2)$ & NR & 100 & 42 & $20.3(4)$ & $45(16)$ & $47(12)$ & \multirow{3}{*}{$\begin{array}{l}\text { RCT, Spain, single center, open } \\
\text { label, } 24 \text { months, funded by } \\
\text { Spanish Health Ministry }\end{array}$} \\
\hline & $\mathrm{CsA}+\mathrm{MMF}+$ Ster & $80(37.5)$ & NR & 100 & 50 & $21.0(4)$ & $42(15)$ & $48(14)$ & \\
\hline & $\mathrm{TAC}+\mathrm{MMF}+$ Ster & $80(45.0)$ & NR & 100 & 59 & $21(4)$ & $44(17)$ & $47(11)$ & \\
\hline \multicolumn{10}{|c|}{ 6. AMETAB vs. AMETAB } \\
\hline \multirow{3}{*}{$\begin{array}{l}\text { 1. Keown } \\
(1995)[49]\end{array}$} & \multirow{3}{*}{$\begin{array}{c}\mathrm{AZA}+\mathrm{CsA}+\text { Pred } \\
\mathrm{MMF}+\mathrm{CsA}+\text { Pred } \\
\mathrm{MMF}(\mathrm{hd})+\mathrm{CsA}+ \\
\text { Pred }\end{array}$} & $173(46.2)$ & NR & 10.4 & NR & $20(7)$ & $38(16)$ & $46(13)$ & \multirow{3}{*}{$\begin{array}{l}\text { RCT, Canada, multicenter (21), } \\
\text { double blind, } 24 \text { months }\end{array}$} \\
\hline & & $166(33.1)$ & NR & 14.46 & NR & $21(9)$ & $39(16)$ & $47(13)$ & \\
\hline & & $164(40.2)$ & NR & 10.98 & NR & $20(7)$ & $37(16)$ & $46(13)$ & \\
\hline \multicolumn{10}{|l|}{ 2. Pescovitz } \\
\hline (1998) [50] & $\mathrm{AZA}+\mathrm{CsA}+\mathrm{Ster}$ & $108(40.7)$ & 68.5 & 87 & NR & NR & NR & $43.7(11.7)$ & \multirow{2}{*}{$\begin{array}{l}\text { RCT, USA, multi centric (15), } \\
\text { double blind, } 12 \text { and } 36 \text { months }\end{array}$} \\
\hline $\begin{array}{l}\text { [Pescovitz } \\
(2001)][51]\end{array}$ & $\mathrm{MMF}+\mathrm{CsA}+\mathrm{Ster}$ & $113(36.3)$ & 21.3 & 91 & NR & NR & NR & $43.1(11.6)$ & \\
\hline \multirow{2}{*}{$\begin{array}{l}\text { 3. Folkmane } \\
\text { (2002) [52] }\end{array}$} & $\mathrm{AZA}+\mathrm{CsA}+$ Pred & 23 & NR & NR & 100 & NR & NR & $43.2(12.1)$ & \multirow{2}{*}{ RCT, Lithonia, 12 months } \\
\hline & $\mathrm{MMF}+\mathrm{CsA}+$ Pred & 23 & NR & NR & 100 & NR & NR & $43.2(12.1)$ & \\
\hline \multirow{2}{*}{$\begin{array}{l}\text { 4. Sadek } \\
(2002)[53]\end{array}$} & $\mathrm{AZA}+\mathrm{CsA}+$ Pred & $157(29.0)$ & 91.4 & 100 & 87 & NR & NR & $43.9(12.8)$ & \multirow{2}{*}{$\begin{array}{l}\text { RCT, United Kingdom, } \\
\text { multicenter ( } 28) \text {, open label, } 12 \\
\text { months, funded by Novartis }\end{array}$} \\
\hline & $\mathrm{MMF}+\mathrm{CsA}+$ Pred & $162(40.1)$ & 90.4 & 100 & 86 & NR & NR & $43.9(13.0)$ & \\
\hline
\end{tabular}


Table 1. Cont.

\begin{tabular}{|c|c|c|c|c|c|c|c|c|c|}
\hline Study (year) & Treatment & $\begin{array}{c}\text { N (female } \\
\%)\end{array}$ & $\begin{array}{l}\text { White } \\
\%\end{array}$ & $\begin{array}{c}\text { First } \\
\text { Transplant } \\
\%\end{array}$ & $\begin{array}{l}\text { Deceased } \\
\text { Donor \% }\end{array}$ & $\begin{array}{l}\text { Cold Ischemia time } \\
\text { (SD) }\end{array}$ & $\begin{array}{l}\text { Mean Donor Age } \\
\text { (SD) }\end{array}$ & $\begin{array}{l}\text { Mean Age } \\
\text { (SD) }\end{array}$ & $\begin{array}{l}\text { Study design, location, time } \\
\text { of follow-up and funding }\end{array}$ \\
\hline \multicolumn{10}{|c|}{ 7. AMETAB vs.TOR-I } \\
\hline $\begin{array}{l}\text { 1. Vitko } \\
(2004)[54] \\
{[\text { Vitko }(2005)]} \\
{[55]}\end{array}$ & $\begin{array}{c}\mathrm{MMF}+\mathrm{CsA} \\
\mathrm{EVR}(\mathrm{hd})+\mathrm{CsA} \\
\mathrm{EVR}(\mathrm{ld})+\mathrm{CsA}\end{array}$ & $\begin{array}{l}194 \\
198 \\
196\end{array}$ & $\begin{array}{l}\text { NR } \\
\text { NR } \\
\text { NR }\end{array}$ & $\begin{array}{l}100 \\
100 \\
100\end{array}$ & $\begin{array}{l}\text { NR } \\
\text { NR } \\
\text { NR }\end{array}$ & & & $\begin{array}{l}\text { NR } \\
\text { NR } \\
\text { NR }\end{array}$ & $\begin{array}{c}\text { RCT, Czech Republic, } \\
\text { multicenter (54), double blind, } \\
12 \text { and } 36 \text { months, funded by } \\
\text { Novartis }\end{array}$ \\
\hline $\begin{array}{l}\text { 2. Lorber } \\
(2005)[56]\end{array}$ & $\begin{array}{c}\text { MMF + CsA } \\
+ \text { Pred } \\
\text { EVR(hd) + Csa } \\
+ \text { Pred } \\
\text { EVR(ld) + CsA } \\
+ \text { Pred }\end{array}$ & $\begin{array}{l}196(32.7) \\
194(36.6) \\
193(29.5)\end{array}$ & $\begin{array}{l}65.8 \\
63.4 \\
70.5\end{array}$ & $\begin{array}{l}100 \\
100 \\
100\end{array}$ & $\begin{array}{l}45.9 \\
51.5 \\
52.3\end{array}$ & $\begin{array}{l}\text { CAD:18.6 (6.42); } \\
\text { LD:1.3 (1.16); } \\
\text { CAD:18.8 (6.43); } \\
\text { LD:1.2 (1.14) } \\
\text { CAD:19.5 (7.18); } \\
\text { LD: } 1.4(3.4) \\
\end{array}$ & $\begin{array}{l}36.7(13.81) \\
38.4(13.66) \\
37.4(13.55)\end{array}$ & $\begin{array}{l}43.4 \\
43.7 \\
43.3\end{array}$ & $\begin{array}{c}\text { RCT, Switzerland, multicenter } \\
\text { (44), } 36 \text { months, funded } \\
\text { by Novartis }\end{array}$ \\
\hline $\begin{array}{l}\text { 3. Mendez } \\
\text { (2005) [57] }\end{array}$ & $\begin{array}{c}\mathrm{MMF}+\mathrm{TAC} \\
+ \text { Pred } \\
\mathrm{SRL}+\mathrm{TAC}+ \\
\text { Pred }\end{array}$ & $\begin{array}{l}176(30.1) \\
185(33.5)\end{array}$ & $\begin{array}{l}54.0 \\
50.8\end{array}$ & $\begin{array}{l}\text { NR } \\
\text { NR }\end{array}$ & $\begin{array}{l}64.2 \\
63.2\end{array}$ & $\begin{array}{l}19.8 \\
19.1\end{array}$ & $\begin{array}{l}\mathrm{NR} \\
\mathrm{NR}\end{array}$ & $\begin{array}{l}47.8(12.3) \\
45.3(12.4)\end{array}$ & $\begin{array}{l}\text { RCT, USA, multicenter } \\
\text { (27),open label, } 12 \text { months, } \\
\text { funded by Fujisawa }\end{array}$ \\
\hline $\begin{array}{l}\text { 4. Sampaio } \\
(2007)[58]\end{array}$ & $\begin{array}{c}\text { MMF + TAC } \\
+ \text { Pred } \\
\text { SRL+TAC+Pred }\end{array}$ & $\begin{array}{l}50(24.0) \\
50(38.0)\end{array}$ & $\begin{array}{l}54.0 \\
42.0\end{array}$ & $\begin{array}{l}100 \\
100\end{array}$ & $\begin{array}{l}24.0 \\
24.0\end{array}$ & $\begin{array}{l}\text { NR } \\
\text { NR }\end{array}$ & $\begin{array}{l}41.9(10.5) \\
41.6(10.0)\end{array}$ & $\begin{array}{l}42.6(14.2) \\
37.4(10.3)\end{array}$ & $\begin{array}{l}\text { RCT, Brazil, single center, open } \\
\text { label, } 12 \text { months, funded by } \\
\text { Janssen-Cilag }\end{array}$ \\
\hline $\begin{array}{l}\text { 5. Tedesco-Silva } \\
\text { (2010) [59]; } \\
\text { Cibrik } \\
\text { (2013) [60] }\end{array}$ & $\begin{array}{c}\text { MMF } \\
\text { EVR } \\
\text { EVR(ld) }\end{array}$ & $\begin{array}{l}277(31.8) \\
279(31.5) \\
277(36.5)\end{array}$ & $\begin{array}{l}68.6 \\
64.5 \\
69.7\end{array}$ & $\begin{array}{l}100 \\
100 \\
100\end{array}$ & $\begin{array}{l}46.2 \\
45.9 \\
46.6\end{array}$ & $\begin{array}{l}\text { NR } \\
\text { NR } \\
\text { NR }\end{array}$ & $\begin{array}{l}41.8(13.6) \\
41.1(13.0) \\
41.4(13.9)\end{array}$ & $\begin{array}{l}47.2(12.7) \\
45.3(13.4) \\
45.7(12.7)\end{array}$ & $\begin{array}{l}\text { RCT, Brazil, multicenter, open } \\
\text { label, } 12 \text { and } 24 \text { months, funded } \\
\text { by Novartis }\end{array}$ \\
\hline \multicolumn{10}{|c|}{ 8. CNI vs. CNI vs. TOR-I } \\
\hline $\begin{array}{l}\text { 1. Ekberg } \\
(2007)[61] ; \\
\text { Ekberg } \\
(2009)[62]\end{array}$ & $\begin{array}{c}\text { CsA(sd) }+ \text { MMF + } \\
\text { Ster } \\
\text { CsA(ld) }+ \text { MMF + } \\
\text { Ster } \\
\text { TAC(ld) }+ \text { MMF + } \\
\text { Ster } \\
\text { SRL(ld) }+ \text { MMF + } \\
\text { Ster }\end{array}$ & $\begin{array}{l}384(37.7) \\
408(33.6) \\
403(34.2) \\
380(33.3)\end{array}$ & $\begin{array}{l}92.1 \\
92.2 \\
94.0 \\
94.2\end{array}$ & $\begin{array}{l}\text { NR } \\
\text { NR } \\
\text { NR } \\
\text { NR }\end{array}$ & $\begin{array}{l}65.6 \\
64.2 \\
62.8 \\
64.2\end{array}$ & $\begin{array}{l}16.6(5.5) \\
16.8(5.2) \\
16.5(5.7) \\
16.0(5.8)\end{array}$ & $\begin{array}{l}44.6(15.9) \\
46.2(15.1) \\
45.2(15.5) \\
46.0(14.8)\end{array}$ & $\begin{array}{l}45.9(13.8) \\
47.2(13.5) \\
45.4(14.7) \\
44.9(14.5)\end{array}$ & $\begin{array}{l}\text { RCT (12 months) and Cohort } \\
\text { ( } 36 \text { months), Sweden, } \\
\text { multicenter (15), open label, } \\
12 \text { and } 36 \text { months, funded by } \\
\text { Hoffman-La Roche }\end{array}$ \\
\hline
\end{tabular}


Table 1. Cont.

\begin{tabular}{|c|c|c|c|c|c|c|c|c|c|}
\hline Study (year) & Treatment & N (female \%) & $\begin{array}{l}\text { White } \\
\%\end{array}$ & $\begin{array}{c}\text { First } \\
\text { Transplant } \\
\% \\
\end{array}$ & $\begin{array}{l}\text { Deceased } \\
\text { Donor \% }\end{array}$ & $\begin{array}{l}\text { Cold Ischemia time } \\
\text { (SD) }\end{array}$ & $\begin{array}{l}\text { Mean Donor Age } \\
\text { (SD) }\end{array}$ & $\begin{array}{l}\text { Mean Age } \\
\text { (SD) }\end{array}$ & $\begin{array}{l}\text { Study design, location, time } \\
\text { of follow-up and funding }\end{array}$ \\
\hline \multicolumn{10}{|c|}{ 9. CNI+AMETAB vs. CNI+TOR-I } \\
\hline $\begin{array}{l}\text { 1.Kumar }{ }^{\dagger} \\
(2005)[63]\end{array}$ & $\begin{array}{c}\mathrm{CsA}+\mathrm{MMF} \\
\mathrm{CsA}+\mathrm{SRL} \\
\mathrm{TAC}+\mathrm{MMF} \\
\mathrm{TAC}+\mathrm{SRL}\end{array}$ & $\begin{array}{l}58 \\
52 \\
50 \\
40 \\
\end{array}$ & $\begin{array}{l}\mathrm{AA}=0 \\
\mathrm{~N}-\mathrm{AA} \\
=89\end{array}$ & $\begin{array}{l}\mathrm{AA}=\mathrm{NR} \\
\mathrm{Non}-\mathrm{AA}= \\
\mathrm{NR}\end{array}$ & $\begin{array}{l}\mathrm{AA}=93 \\
\mathrm{n}-\mathrm{AA}=83\end{array}$ & $\begin{array}{l}\mathrm{AA}=15.5(6.8) \\
\mathrm{n}-\mathrm{AA}=15.9(12.1)\end{array}$ & $\begin{array}{l}\mathrm{AA}=42.0(16.5) \\
\mathrm{N}-\mathrm{AA}=42.3 \\
(19.2)\end{array}$ & $\begin{array}{l}\mathrm{AA}=52.9 \\
(12.0) \\
\mathrm{n}-\mathrm{AA}= \\
53.0(15.6)\end{array}$ & $\begin{array}{l}\text { RCT, USA, } \\
\text { single center, } 12 \text { months }\end{array}$ \\
\hline \multicolumn{10}{|c|}{ 10. TOR-I vs. CNI+TOR-I } \\
\hline $\begin{array}{l}\text { 1. Tedesco- } \\
\text { Silva } \\
(2010)[64]\end{array}$ & $\begin{array}{l}\mathrm{SRL} \\
\mathrm{SRL}+\mathrm{CsA}\end{array}$ & $\begin{array}{l}102(36.3) \\
105(36.2)\end{array}$ & $\begin{array}{l}72.6 \\
62.9\end{array}$ & $\begin{array}{l}98.0 \\
98.1\end{array}$ & $\begin{array}{l}31.4 \\
30.5\end{array}$ & $\begin{array}{l}7.36(0.99) \\
7.64(1.03)\end{array}$ & $\begin{array}{l}\text { NR } \\
\text { NR }\end{array}$ & $\begin{array}{l}41.5 \\
40.9\end{array}$ & $\begin{array}{l}\text { RCT, Brazil, multicenter (9), } \\
\text { open label, } 12 \text { months, funded } \\
\text { by Wyeth }\end{array}$ \\
\hline \multicolumn{10}{|c|}{ 11. CNI+AMETAB vs. CNI vs. AMETAB } \\
\hline $\begin{array}{l}\text { 1. Gheith } \\
\text { (2008) [69] }\end{array}$ & $\begin{array}{l}\mathrm{CsA}+\mathrm{AZA}+\text { Pred } \\
\mathrm{CsA}+\text { Pred } \\
\mathrm{AZA}+\text { Pred }\end{array}$ & $\begin{array}{l}239(26.36) \\
75(42.67) \\
130(26.92) \\
\end{array}$ & $\begin{array}{l}\mathrm{NR} \\
\mathrm{NR} \\
\mathrm{NR}\end{array}$ & $\begin{array}{l}\text { NR } \\
\text { NR } \\
\text { NR }\end{array}$ & $\begin{array}{l}0 \\
0 \\
0\end{array}$ & $\begin{array}{l}\text { NR } \\
\text { NR } \\
\text { NR }\end{array}$ & $\begin{array}{l}34.0(9.2) \\
34.6(10.3) \\
33.3(10.1)\end{array}$ & $\begin{array}{l}30.7(10.1) \\
28.1(10.3) \\
29.8(7.9) \\
\end{array}$ & $\begin{array}{l}\text { Cohort, Egypt, single Center, } \\
20 \text { years }\end{array}$ \\
\hline \multicolumn{10}{|c|}{ 12. TOR-I + CNI-Elim vs. TOR-I vs. CNI } \\
\hline $\begin{array}{l}\text { 1. Flechner } \\
(2011)[65]\end{array}$ & $\begin{array}{l}\text { SRL + TAC-Elim } \\
\text { SRL + MMF } \\
\text { TAC + MMF }\end{array}$ & $\begin{array}{l}152(28.3) \\
152(27.6) \\
139(41.7)\end{array}$ & $\begin{array}{l}75.0 \\
77.0 \\
73.4\end{array}$ & $\begin{array}{l}92.8 \\
91.5 \\
92.1\end{array}$ & $\begin{array}{l}60.5 \\
63.2 \\
64.0\end{array}$ & $\begin{array}{l}17.7(6.7) \\
17.3(5.7) \\
17.4(6.3)\end{array}$ & $\begin{array}{l}43.2(13.6) \\
45.5(14.9) \\
44.4(13.9)\end{array}$ & $\begin{array}{l}47.9(13.3) \\
50.4(13.0) \\
48.4(13.2)\end{array}$ & $\begin{array}{l}\text { RCT, USA, multicenter ( } 65) \text {, } \\
\text { open-label, } 24 \text { months, funded } \\
\text { by Wyeth }\end{array}$ \\
\hline
\end{tabular}

Abbreviations: AMETAB, Antimetabolites; CAD, Cadaveric donor; Elim, elimination; LD, Living donor; NHBD, Non-heart beating donor; NR, not reported; Pred, Prednisone; Ster, Steroids; (ld), Low dose; (sd), Standard dose; (hd), High dose. ${ }^{\dagger}$ The study compares AA (African American) and n-AA (non-African American) recipients 


\subsection{Outcomes}

All adverse events reported in the included articles were collected, and the most prevalent events were included in the synthesis. The following outcomes were included: abdominal pain, anemia, bacterial infections (all definitions), cytomegalovirus (CMV) infections, diabetes mellitus (new-onset diabetes mellitus, post-transplant diabetes, and use of hypoglycemic drugs were considered), diarrhea, dyslipidemia (hypercholesterolemia, hypertriglyceridemia and hyperlipidemia were considered), gastritis, total infections (as reported in the study), hypertension (use of antihypertensive drugs was also considered), leukopenia, lymphoceles, malignancies (all types), nausea, vomiting, thrombocytopenia, urinary tract infection (UTI) and withdrawal (discontinuation and crossover of study medication were considered).

For data synthesis and analysis, the comparable schemes in each study were classified into the following groups: CNI vs. CNI; AMETAB vs. AMETAB;TOR-I vs. CNI; CNI + AMETAB vs. CNI; TOR-I vs. AMETAB; and AMETAB vs. CNI. In some studies, it was possible to compare more than one group, such as studies that included the treatment protocol of CNI + AMETAB vs. CNI + AMETAB vs. $\mathrm{CNI}+\mathrm{AMETAB}$ (it was possible to compare CNI vs. CNI and AMETAB vs. AMETAB).

\subsubsection{CNI vs. CNI}

All studies that compared CsA and TAC were included in this group. A total of 17 articles $[22-32,48,61,62,66-68]$ reported safety data related to TAC as the experimental treatment and CsA as the control. One study used low-dose TAC (3-7 ng/mL) and low-dose CsA $(50-100 \mathrm{ng} / \mathrm{mL})[61,62]$, whereas the others used standard doses of both drugs $(5-15 \mathrm{ng} / \mathrm{mL}$ for TAC and $150-300 \mathrm{ng} / \mathrm{mL}$ for CsA).

The results of 13 articles, two cohorts and 11 RCTs, were meta-analyzed and are displayed in Table 2. Both the cohort and RCT pooled results indicate that TAC was associated with an increased risk for diabetes (Figure 3). This association was also found at 120 months follow-up in one cohort that was not included in the pooled analysis $(n=192 ; \mathrm{RR}=2.10 ; 95 \% \mathrm{CI}: 1.17,3.77 ; p=0.01)$ [67]. The risk of dyslipidemia was reduced in TAC regimens, as shown in the meta-analysis and in two single studies: a cohort of 36 months $(\mathrm{n}=506$; $\mathrm{RR}=0.74 ; 95 \% \mathrm{CI}: 0.57,0.97 ; p=0.03)$ [62] and a RCT of 60 months $(n=76 ; \mathrm{RR}=0.62 ; 95 \% \mathrm{CI}: 0.40,0.95 ; p=0.03)$ [31].

Although the other outcomes had no statistical significance in the pooled results, studies in single analysis showed that TAC was associated with a higher risk of withdrawing the treatment at 120 months of follow-up $(\mathrm{n}=192 ; \mathrm{RR}=11.21 ; 95 \% \mathrm{CI}: 2.50,50.23 ; p=0.002)$ [67] and that CsA presented a greater risk for hypertension at 36 months of follow-up $(n=89 ; \mathrm{RR}=0.67 ; 95 \% \mathrm{CI}: 0.48$, $0.94 ; p=0.02$ ) [26]. 
Table 2. Meta-analysis results of outcomes reported by studies comparing TAC vs. CsA a .

\begin{tabular}{clcccc}
\hline \multirow{2}{*}{ Outcome } & \multicolumn{1}{c}{ Study Design (N) } & \multirow{2}{*}{ Time in months } & $\begin{array}{c}\text { Relative Risk } \\
\text { b }\end{array}$ & & \multicolumn{2}{c}{ Statistics $^{\mathbf{c}}$} \\
\cline { 5 - 6 } & & & $\mathbf{9 5 \%}$ CI) & $\boldsymbol{p}$ & $\mathbf{I}^{\mathbf{2}}$ \\
\hline CMV & RCT $[23,24,29,61](1519)$ & 12 & $0.85(0.64,1.15)$ & 0.30 & 0 \\
Diabetes & RCT $[22-25,27-30,32,61](2389)$ & 36 & $2.71(1.61,4.57)$ & 0.0002 & 0 \\
& Cohort $[62,66](738)$ & 12 & $0.75(0.60,0.94)$ & 0.01 & 0 \\
Dyslipidemia & RCT $[29,30,61](1435)$ & 12 & $0.97(0.82,1.16)$ & 0.76 & 25 \\
Hypertension & RCT $[23,26,27,29,61](1714)$ & 12 & $1.03(0.93,1.14)$ & 0.55 & 12 \\
Total Infections & RCT $[23-25,61](1376)$ & 12 & $0.61(0.34,1.07)$ & 0.09 & 10 \\
Lymphoceles & RCT $[30,61](1235)$ & 12 & $1.16(0.40,3.38)$ & 0.79 & 0 \\
Malignancies & RCT $[23,29,61](1459)$ & 12 & $0.98(0.34,2.81)$ & 0.97 & $82 *$ \\
Withdraw & RCT $[23,24,27-30,32,61](2384)$ &
\end{tabular}

${ }^{\mathrm{a}}$ Results reaching statistical significance are in bold font. ${ }^{\mathrm{b}}$ Relatives risk values of $<1$ favor treatment with TAC. ${ }^{c} p$ : $p$-value for relative risk estimation; $\mathrm{I}^{2}$ : test for heterogeneity. ${ }^{*}$ The high heterogeneity $(p<0.00001)$ could be caused by the following trials: Mayer 1997 [23], Hardinger 2005 [29] and Vicenti 1996 [32]. Sensitivity analysis showed much reduced heterogeneity $(p=0.23, \mathrm{I} 2=29 \%)$ when these trials were removed from the analysis.

Figure 3. Meta-analysis of diabetes for TAC vs. CsA comparison at 12 and 36 months.

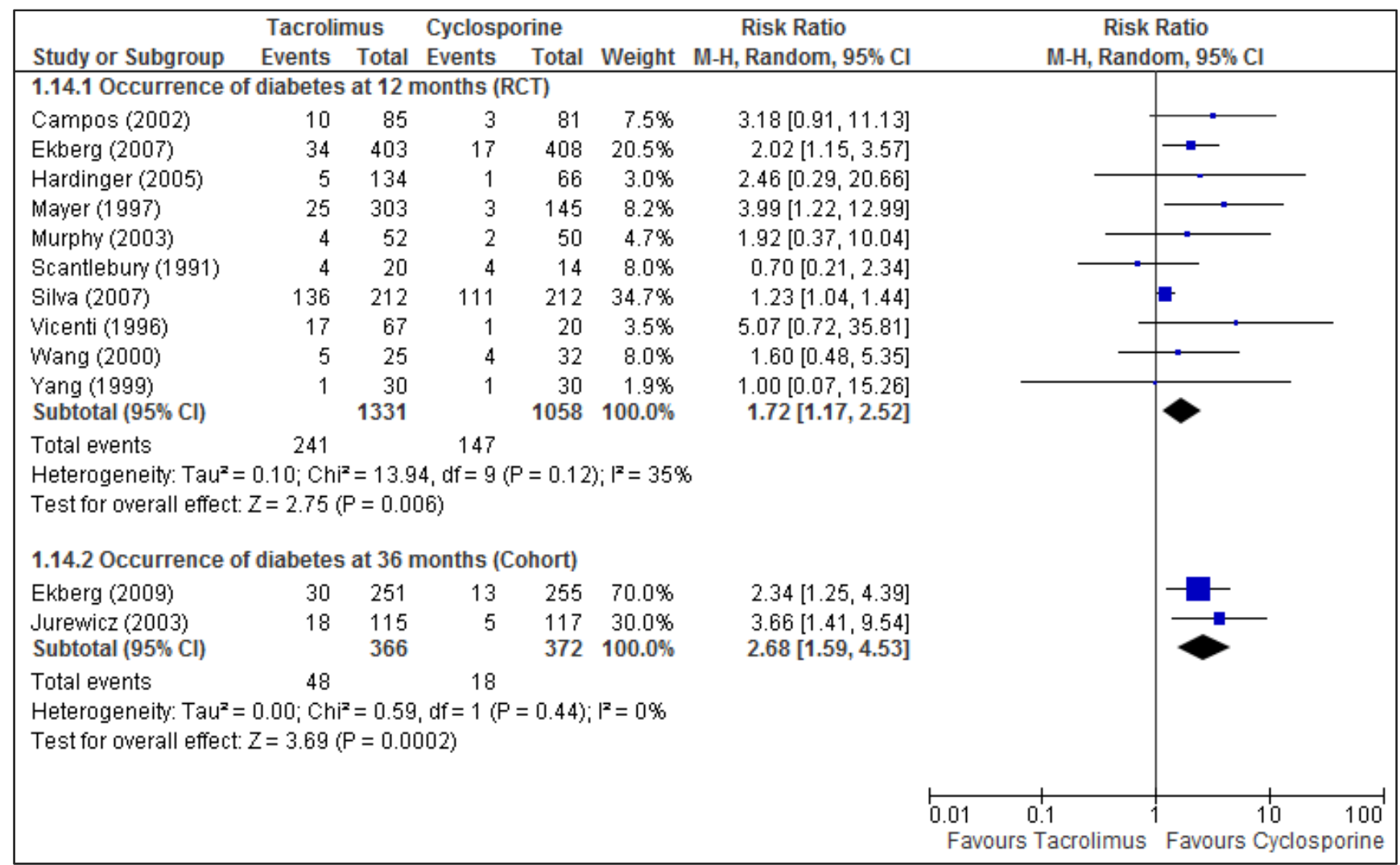

* The study of Ekberg is a RCT with 12 months of experimental data and 36 months of observational data, so that the article containing results at 36 months results was included in cohort analysis. 


\subsubsection{AMETAB vs. AMETAB}

Five articles from four RCTs with 12 months of follow-up compared AZA (control) with MMF (intervention) [49-53]. The dosage used in the studies ranged from 100-150 mg/day for AZA and 2-3 g/day for MMF.

All five articles were included in meta-analysis and the results are shown in Table 3. MMF was associated with an increased risk for total infections and gastrointestinal discomfort, including abdominal pain, diarrhea and vomiting. The sensitivity analysis for heterogeneity indicated that participants who were taking MMF had a higher risk of withdrawing from the treatment.

Table 3. Meta-analysis results of outcomes reported by studies comparing MMF vs. AZA a .

\begin{tabular}{llccccc}
\hline \multirow{2}{*}{ Outcome } & \multirow{2}{*}{ Study Design (N) } & \multirow{2}{*}{ Time in months } & $\begin{array}{c}\text { Relative Risk }^{\mathbf{b}} \\
\mathbf{( 9 5 \%} \mathbf{C I})\end{array}$ & \multicolumn{2}{c}{ Statistics $^{\mathbf{c}}$} \\
\hline Total Infections & RCT $[49,51-53](919)$ & 12 & $1.17(1.03,1.33)$ & 0.01 & 0 \\
CMV & RCT $[49,51-53](919)$ & 12 & $0.94(0.82,1.03)$ & 0.17 & 41 \\
Abdominal pain & RCT $[49,50,53](873)$ & 12 & $1.40(1.06,1.83)$ & 0.02 & 14 \\
Diarrhea & RCT $[49,50,53](873)$ & 12 & $1.49(1.17,1.90)$ & 0.001 & 10 \\
Nausea & RCT $[49,50,53](873)$ & 12 & $0.98(0.69,1.39)$ & 0.91 & 41 \\
Vomiting & RCT $[49,50,53](873)$ & 12 & $1.54(1.10,2.15)$ & 0.01 & 0 \\
Malignancies & RCT $[49,53](652)$ & 12 & $1.52(0.81,2.82)$ & 0.19 & 0 \\
Withdraw & RCT $[49,50,53](873)$ & 12 & $1.21(0.77,1.92)$ & 0.40 & $66^{*}$ \\
\hline
\end{tabular}

${ }^{a}$ Results reaching statistical significance are in bold font. ${ }^{\mathrm{b}}$ Relatives risk values of $<1$ favor treatment with MMF. ${ }^{c} p$ : $p$-value for relative risk estimation; $\mathrm{I}^{2}$ : test for heterogeneity. ${ }^{*} \mathrm{RCT}$ Sadek 2002 [53] is largely responsible for the heterogeneity among RCTs that reported withdraw. Sensitivity analysis showed a relative risk of 1.93 (1.06 to 3.52), and dramatically reduced heterogeneity ( $p=0.95, \mathrm{I}^{2}=0 \%$ ) when this trial was removed from the analysis.

\subsubsection{TOR-I vs. CNI}

Eight articles from five RCTs with 12 months of follow-up and one RCT of 24 months were included in this group: two accessing SRL vs. TAC [47,65], four accessing SRL vs. CsA [43-46] and two accessing both [61,62]. In these studies, SRL was used at low $(4-8 \mathrm{ng} / \mathrm{mL})$ and standard (10-20ng/mL) doses, as well as CsA, whereas TAC was administered only at low dose. SRL was applied as experimental drug and CsA and TAC served as controls.

Five articles were included in the meta-analysis (Table 4). When compared with any of the CNIs, the use of SRL presented a higher risk for anemia, dyslipidemia, lymphoceles and withdrawal. The association between SRL and anemia was also reported by one cohort of 36 months, which was not included in the pooled analysis, compared with CsA $(\mathrm{n}=476 ; \mathrm{RR}=1.30 ; 95 \% \mathrm{CI}: 1.05,1.60 ; p=0.02)$ and TAC ( $\mathrm{n}=472 ; \mathrm{RR}=1.29 ; 95 \% \mathrm{CI}: 1.05,1.60 ; p=0.02)$ [62]. A similar result was observed for dyslipidemia when SRL was compared with TAC $(n=472 ; \mathrm{RR}=1.42 ; 95 \% \mathrm{CI}$ : 1.15, 1.76; $p=0.001$ ) [62]. Single analysis of a RCT comparing SRL and TAC at 24 months showed similar results with the SRL vs. TAC meta-analysis: SRL was associated with increased risk of anemia, dyslipidemia, lymphoceles and withdrawal, and had no difference for malignancy, infections or 
hypertension [65]. Moreover, the risk of diabetes was higher for SRL, and the risk of CMV infection was higher for CsA.

Table 4. Meta-analysis results of outcomes reported by studies comparing SRL vs. CsA or TAC ${ }^{\mathrm{a}}$.

\begin{tabular}{|c|c|c|c|c|c|c|c|c|c|c|}
\hline \multirow[b]{3}{*}{ Outcome } & \multicolumn{5}{|c|}{ SRL vs. CsA } & \multicolumn{5}{|c|}{ SRL vs. TAC } \\
\hline & \multirow[b]{2}{*}{$\begin{array}{l}\text { Study Design } \\
\text { (N) }\end{array}$} & \multirow[b]{2}{*}{$\begin{array}{l}\text { Time } \\
\text { (mo.) }\end{array}$} & \multirow{2}{*}{$\begin{array}{c}\text { Relative Risk }^{\text {b }} \\
\text { (95\% CI) }\end{array}$} & \multicolumn{2}{|c|}{ Statistics $^{c}$} & \multirow[b]{2}{*}{$\begin{array}{c}\text { Study } \\
\text { Design (N) }\end{array}$} & \multirow[b]{2}{*}{$\begin{array}{l}\text { Time } \\
\text { (mo.) }\end{array}$} & \multirow[b]{2}{*}{$\begin{array}{c}\text { Relative Risk }^{\text {b }} \\
\text { (95\% CI) }\end{array}$} & \multicolumn{2}{|c|}{ Statistics $^{c}$} \\
\hline & & & & $p$ & $\mathbf{I}^{2} \%$ & & & & $p$ & $\begin{array}{l}\mathrm{I}^{2} \\
\%\end{array}$ \\
\hline $\begin{array}{l}\text { Total } \\
\text { Infections }\end{array}$ & $\begin{array}{l}\text { RCT }[46,61] \\
(927)\end{array}$ & 12 & $\begin{array}{c}0.98 \\
(0.82,1.18)\end{array}$ & 0.86 & 33 & - & - & - & - & - \\
\hline CMV & $\begin{array}{l}\text { RCT } \\
{[43,44,46,61]} \\
(1,155)\end{array}$ & 12 & $\begin{array}{c}0.46 \\
(0.25,0.85)\end{array}$ & 0.01 & $53^{d}$ & $\begin{array}{l}\mathrm{RCT}[47,61] \\
(924)\end{array}$ & 12 & $0.26(0.03,2.30)$ & 0.23 & 79 \\
\hline UTI & $\begin{array}{l}\mathrm{RCT} \\
{[43,44,46,61]} \\
(1,155)\end{array}$ & 12 & $\begin{array}{c}1.04 \\
(0.79,1.37)\end{array}$ & 0.79 & 35 & - & - & - & - & - \\
\hline Anemia & $\begin{array}{l}\mathrm{RCT}[43,46,61] \\
(1,010)\end{array}$ & 12 & $\begin{array}{c}1.48 \\
(1.16,1.90)\end{array}$ & $<0.01$ & 0 & $\begin{array}{l}\mathrm{RCT}[47,61] \\
(924)\end{array}$ & 12 & $1.56(1.26,1.93)$ & $<0.01$ & 0 \\
\hline Leukopenia & $\begin{array}{l}\mathrm{RCT}[43,46,61] \\
(1,010)\end{array}$ & 12 & $\begin{array}{c}1.32 \\
(0.70,2.47)\end{array}$ & 0.39 & $57^{\mathrm{e}}$ & $\begin{array}{l}\mathrm{RCT}[47,61] \\
(924)\end{array}$ & 12 & $0.82(0.59,1.14)$ & 0.24 & 0 \\
\hline Dyslipidemia & $\begin{array}{l}\mathrm{RCT}[43,46,61] \\
(1,010)\end{array}$ & 12 & $\begin{array}{c}2.02 \\
(1.03,3.97)\end{array}$ & 0.04 & $65^{f}$ & $\begin{array}{l}\text { RCT }[47,61] \\
(924)\end{array}$ & 12 & $1.58(1.10,2.26)$ & 0.01 & 0 \\
\hline Diabetes & $\begin{array}{l}\text { RCT } \\
{[43,44,46,61]} \\
(1,155)\end{array}$ & 12 & $\begin{array}{c}1.82 \\
(1.14,2.89)\end{array}$ & 0.05 & 0 & $\begin{array}{l}\mathrm{RCT}[47,61] \\
(924)\end{array}$ & 12 & $0.78(0.52,1.17)$ & 0.23 & 0 \\
\hline Hypertension & $\begin{array}{l}\text { RCT }[43,46,61] \\
(1,010)\end{array}$ & 12 & $\begin{array}{c}0.94 \\
(0.66,1.33)\end{array}$ & 0.71 & 28 & $\begin{array}{l}\mathrm{RCT}[47,61] \\
(924)\end{array}$ & 12 & $1.53(0.55,4.23)$ & 0.41 & 93 \\
\hline Lymphoceles & $\begin{array}{l}\mathrm{RCT}[44,46,61] \\
(1,072)\end{array}$ & 12 & $\begin{array}{c}1.65 \\
(1.10,2.46)\end{array}$ & 0.01 & 18 & $\begin{array}{l}\text { RCT }[47,61] \\
(924)\end{array}$ & 12 & $2.92(1.73,4.93)$ & $<0.01$ & 0 \\
\hline Malignancies & $\begin{array}{l}\mathrm{RCT}[43,61] \\
(871)\end{array}$ & 12 & $\begin{array}{c}1.09 \\
(0.09,13.46)\end{array}$ & 0.95 & 60 & - & - & - & - & - \\
\hline Withdraw & $\begin{array}{l}\text { RCT } \\
{[43,44,46,61]} \\
(1,155)\end{array}$ & 12 & $\begin{array}{c}3.68 \\
(2.22,6.11)\end{array}$ & $<0.01$ & 0 & $\begin{array}{l}\mathrm{RCT}[47,61] \\
(924)\end{array}$ & 12 & $4.31(2.32,7.99)$ & $<0.01$ & 0 \\
\hline
\end{tabular}

Abbreviations: UTI, urinary tract infection. ${ }^{a}$ Results reaching statistical significance are in bold font. ${ }^{\mathrm{b}}$ Relatives risk values of $<1$ favor treatment with SRL. ${ }^{c} p$ : $p$-value for relative risk estimation; $\mathrm{I}^{2}$ : test for heterogeneity. ${ }^{\mathrm{d}}$ Sensitivity analysis removing Groth 1998 [43]: RR 0.38 (0.21 to $\left.0.67 ; \mathrm{I}^{2}=38 \%\right)$. ${ }^{\mathrm{e}}$ Sensitivity analysis removing Groth 1998 [43]: RR 1.0 (0.69 to 1.47; $\left.\mathrm{I}^{2}=0 \%\right) .{ }^{\mathrm{f}}$ Sensitivity analysis removing Ekberg 2007 [61]: RR 3.01 (1.61 to 5.62; $\left.\mathrm{I}^{2}=0 \%\right)$.

Lebranchu et al. (2012) reported the 5-years results of the RCT from Buchler et al. [44], which compared SRL vs. CsA, and reported outcomes as mouth ulcers, acne, UTI, malignancies, diabetes and withdrawal due to adverse event, but none showed difference between groups in single analysis [45].

One RCT with 12 months of follow-up compared SRL in combination with CsA vs. SRL alone [64] and reported anemia, hypertension, CMV infection, lymphoceles, dyslipidemia, diabetes and polycythemia; however, only polycythemia was associated with the combination of TOR-I and CNI $(\mathrm{n}=207 ; \mathrm{RR}=3.40 ; 95 \%$ CI: $1.16,9.98 ; p=0.03)$. 


\subsubsection{CNI + AMETAB vs. CNI}

Seven studies were included in this group: one cohort with 240 months of follow-up comparing CsA+AZA vs. CsA [69], one RCT with 12 months of follow-up comparing CsA + MMF vs. CsA [33], three RCTs with 12 and 36 months of follow-up comparing TAC + AZA vs. TAC [36-38], one RCT with 12 months of follow-up comparing TAC + MMF vs. TAC [39] and one RCT with 12 months of follow-up comparing CsA + AZA vs. TAC [34]. CsA and MMF were administrated at both low and standard doses, whereas TAC and AZA were used only at the standard dose.

Two studies comparing TAC + AZA vs. TAC at 12 months of follow-up were meta-analyzed and the results are displayed on Table 5. The meta-analysis revealed that the combination of TAC and AZA was associated with a greater risk of leukopenia and withdrawal compared with TAC alone. The RCT with 36 months of follow-up confirmed this result for leukopenia $(n=475$; RR $=5.60 ; 95 \%$ CI: $2.39,13.08 ; p<0.01)$ [37]. In a single analysis, the combination of TAC and AZA was associated with anemia at 12 months of follow-up $(\mathrm{n}=475 ; \mathrm{RR}=1.55 ; 95 \% \mathrm{CI}: 1.06,2.28 ; p=0.02)$ [36].

Table 5. Meta-analysis results of outcomes reported by studies comparing TAC + AZA vs. TAC ${ }^{\text {a }}$.

\begin{tabular}{llcccc}
\hline \multirow{2}{*}{ Outcome } & Study Design (N) & Time in months & $\begin{array}{c}\text { Relative Risk } \\
\text { (95\% CI) }\end{array}$ & \multicolumn{2}{c}{ Statistics $^{\mathbf{c}}$} \\
\cline { 5 - 7 } & & 12 & $0.99(0.82,1.20)$ & 0.94 & 0 \\
Total Infections & RCT [36,38] (720) & 12 & $8.41(3.36,21.02)$ & $<0.01$ & 0 \\
Leukopenia & RCT [36,38] (720) & 12 & $0.85(0.41,1.76)$ & 0.67 & 0 \\
Diabetes mellitus & RCT [36,38] (720) & 12 & $0.83(0.65,1.06)$ & 0.13 & 0 \\
Hypertension & RCT [36,38] (720) & 12 & $0.96(0.68,1.35)$ & 0.82 & 0 \\
Tremor & RCT [36,38] (720) & 12 & $10.39(4.40,24.56)$ & $<0.01$ & 0 \\
Withdraw & RCT [36,38] (720) & 12 &
\end{tabular}

${ }^{\mathrm{a}}$ Results reaching statistical significance are in bold font. ${ }^{\mathrm{b}}$ Relatives risk values of $<1$ favor treatment with Antiproliferative Agent + TAC. ${ }^{c} p$ : $p$-value for relative risk estimation; $\mathrm{I}^{2}$ : test for heterogeneity

The combination of TAC and MMF at 12 months was associated with a greater risk of gastritis $(\mathrm{n}=135 ; \mathrm{RR}=1.92 ; 95 \% \mathrm{CI}: 1.18,3.14 ; p=0.009)$ and leukopenia $(\mathrm{n}=153 ; \mathrm{RR}=3.00 ; 95 \%$ CI: $1.13,8.01 ; p=0.03$ ) [39], compared with TAC alone.

The combination of CsA and AZA at 240 months of follow-up was associated with higher risk for hypertension $(\mathrm{n}=314 ; \mathrm{RR}=1.23 ; 95 \% \mathrm{CI}: 1.04,1.46 ; p=0.02)$ and a lower risk for bacterial infections ( $\mathrm{n}=314$; $\mathrm{RR}=0.31 ; 95 \% \mathrm{CI}: 0.13,0.76 ; p=0.01)$, compared to CsA alone [69]. The studies comparing CsA + MMF vs. CsA and TAC + MMF vs. TAC reported CMV infection, diabetes, diarrhea and leukopenia but the estimated RRs were not significant.

\subsubsection{TOR-I vs. AMETAB}

Seven RCTs were included in this group: four comparing EVL vs. MMF, at 12 months [54,59], 24 months [60], and 36 months of follow-up [55,56] and three comparing SRL vs. MMF at 12 months [57,58] and 24 months of follow-up [63]. SRL and MMF were used at standard doses, and EVL was used at low (1.5 mg/day) and high ( $3 \mathrm{mg} /$ day) doses.

The studies comparing EVL and MMF were meta-analyzed in subgroups of 12 and 36 months of follow-up and divided based on low- and high-dose EVL (Table 6). Additionally, the studies 
comparing SRL and MMF at 12 months of follow-up were meta-analyzed. No differences were observed when comparing low- and high-dose EVL. Independent of dosing, EVL was associated with an increased risk of dyslipidemia and withdrawal. MMF presented a higher risk of CMV infection when compared with both doses of EVL. The single analysis for 24 months of follow-up showed similar results: both doses of EVL were associated with increased risk of withdrawal and lower risk of CMV infection and leukopenia when compared with MMF [60]. In the same study, high-dose EVL was associated with a greater risk of diabetes $(n=833 ; \mathrm{RR}=1.96 ; 95 \% \mathrm{CI}: 1.18,1.87 ; p=0.01)$ [60]. Compared with MMF, SRL showed increased risk of withdrawal in meta-analysis and no significant results were found in single-analysis.

\subsubsection{AMETAB vs. CNI}

Three studies compared an antimetabolite with a CNI: one RCT with 63 months of follow-up that compared MMF vs. TAC [39] and two studies comparing AZA vs. CsA, a cohort with 240 months of follow-up [69] and a RCT with 36 months of follow-up [40]. All drugs were used at standard doses.

There was not a sufficient number of studies with identical follow-up periods to perform meta-analysis. The risk for dyslipidemia $(\mathrm{n}=132 ; \mathrm{RR}=1.75 ; 95 \% \mathrm{CI}: 1.13,2.71 ; p=0.01)$ and diarrhea $(\mathrm{n}=132 ; \mathrm{RR}=3.87 ; 95 \% \mathrm{CI}: 1.35,11.03 ; p=0.01)$ was higher for MMF, compared with $\mathrm{TAC}$, and MMF presented a lower risk of withdrawal than TAC $(\mathrm{n}=132 ; \mathrm{RR}=0.14 ; 95 \% \mathrm{CI}: 0.04$, $0.45 ; p=0.0009)$ [39].

AZA was associated with an increased risk of pulmonary infections $(\mathrm{n}=276$; $\mathrm{RR}=2.25 ; 95 \% \mathrm{CI}$ : $1.01,5.00 ; p=0.05)$ and leukopenia $(\mathrm{n}=276 ; \mathrm{RR}=2.76 ; 95 \% \mathrm{CI}: 1.86,4.08 ; p<0.01)$ [40] and a lower risk of hypertension $(\mathrm{n}=205 ; \mathrm{RR}=0.77 ; 95 \% \mathrm{CI}: 0.61,0.97 ; p=0.03)$ [69].

\section{Conclusions}

Six different groups of immunosuppressant drugs were evaluated and compared. Evaluating the safety of immunosuppressive drugs is complex because kidney transplantation requires the simultaneous use of multiple classes of drugs at varying doses.

The majority of the studies included here showed a low risk of bias, and only one study revealed a high risk of bias for allocation order generation and allocation confidentiality [34]. Based on the parameters described in the Cochrane Handbook [19], the quality of most studies was compromised by a lack of sufficient information to judge the randomization and allocation concealment.

However, this quality assessment did not invalidate the results of the meta-analysis. Overall, the heterogeneity of the treatment-efficacy results was low, indicating small inter-study variability. In general, the observational studies did not show selection bias, and the majority of these studies used the same time in and out of treatment, allowing for a comparison of populations. 
Table 6. Meta-analysis results of outcomes reported by studies comparing EVL or SRL vs. MMF ${ }^{\text {a }}$.

\begin{tabular}{|c|c|c|c|c|c|c|c|c|c|c|c|c|c|c|c|}
\hline \multirow{3}{*}{ Outcome } & \multicolumn{5}{|c|}{ EVL (ld) vs. MMF } & \multicolumn{5}{|c|}{ EVL (hd) vs. MMF } & \multicolumn{5}{|c|}{ SRL vs. MMF } \\
\hline & \multirow{2}{*}{$\begin{array}{c}\text { Study } \\
\text { Design (N) }\end{array}$} & \multirow{2}{*}{$\begin{array}{l}\text { Time } \\
\text { (mo.) }\end{array}$} & \multirow{2}{*}{$\begin{array}{c}\text { Relative Risk }^{\mathbf{b}} \\
(95 \% \mathrm{CI}) \\
\end{array}$} & \multicolumn{2}{|c|}{ Statistics $^{c}$} & \multirow{2}{*}{$\begin{array}{c}\text { Study } \\
\text { Design (N) }\end{array}$} & \multirow{2}{*}{$\begin{array}{l}\text { Time } \\
\text { (mo.) }\end{array}$} & \multirow{2}{*}{$\begin{array}{c}\text { Relative Risk }^{\mathbf{b}} \\
(95 \% \mathrm{CI}) \\
\end{array}$} & \multicolumn{2}{|c|}{ Statistics $^{c}$} & \multirow{2}{*}{$\begin{array}{c}\text { Study } \\
\text { Design (N) }\end{array}$} & \multirow{2}{*}{$\begin{array}{l}\text { Time } \\
\text { (mo.) }\end{array}$} & \multirow{2}{*}{$\begin{array}{c}\text { Relative Risk }^{\mathbf{b}} \\
\text { (95\% CI) } \\
\end{array}$} & \multicolumn{2}{|c|}{ Statistics } \\
\hline & & & & $p$ & $\mathbf{I}^{2}$ & & & & $p$ & $\mathbf{I}^{2}$ & & & & $p$ & $\mathbf{I}^{2}$ \\
\hline Total Infections & $\begin{array}{l}\mathrm{RCT}[54,59] \\
(946)\end{array}$ & 12 & $\begin{array}{c}0.62 \\
(0.26,1.48)\end{array}$ & 0.28 & 92 & $\begin{array}{l}\mathrm{RCT}[54,59] \\
(946)\end{array}$ & 12 & $\begin{array}{c}0.83 \\
(0.58,1.18)\end{array}$ & 0.29 & 70 & - & - & - & - & - \\
\hline \multirow{2}{*}{ CMV infections } & $\begin{array}{l}\text { RCT [54,59] } \\
(946)\end{array}$ & 12 & $\begin{array}{c}0.23 \\
(0.12,0.42)\end{array}$ & $<0.01$ & 0 & $\begin{array}{l}\text { RCT }[54,59] \\
(946)\end{array}$ & 12 & $\begin{array}{c}0.15 \\
(0.01,2.17)\end{array}$ & 0.16 & 73 & \multirow{2}{*}{-} & \multirow{2}{*}{-} & \multirow{2}{*}{-} & \multirow{2}{*}{-} & \multirow{2}{*}{-} \\
\hline & $\begin{array}{l}\text { RCT }[55,56] \\
(781)\end{array}$ & 36 & $\begin{array}{c}0.47 \\
(0.16,1.41)\end{array}$ & 0.18 & 78 & $\begin{array}{l}\mathrm{RCT}[55,56] \\
(780)\end{array}$ & 36 & $\begin{array}{c}0.47 \\
(0.29,0.74)\end{array}$ & $<0.01$ & 0 & & & & & \\
\hline \multirow{2}{*}{ Anemia } & $\begin{array}{l}\text { RCT }[54,59] \\
(946)\end{array}$ & 12 & $\begin{array}{c}0.97 \\
(0.79,1.20)\end{array}$ & 0.80 & 0 & $\begin{array}{l}\mathrm{RCT}[54,59] \\
(946)\end{array}$ & 12 & $\begin{array}{c}1.15 \\
(0.95,1.40)\end{array}$ & 0.15 & 0 & \multirow{2}{*}{-} & \multirow{2}{*}{-} & \multirow{2}{*}{-} & \multirow{2}{*}{-} & \multirow{2}{*}{-} \\
\hline & $\begin{array}{l}\mathrm{RCT}[55,56] \\
(781)\end{array}$ & 36 & $\begin{array}{c}1.17 \\
(0.73,1.88)\end{array}$ & 0.50 & 76 & $\begin{array}{l}\mathrm{RCT}[55,56] \\
(780)\end{array}$ & 36 & $\begin{array}{c}1.47 \\
(0.97,2.23)\end{array}$ & 0.07 & 74 & & & & & \\
\hline Leukopenia & $\begin{array}{l}\text { RCT }[55,56] \\
(781)\end{array}$ & 36 & $\begin{array}{c}0.50 \\
(0.24,1.06)\end{array}$ & 0.07 & 42 & - & - & - & - & - & - & - & - & - & - \\
\hline Dyslipidemia & $\begin{array}{l}\text { RCT [54,59] } \\
(946)\end{array}$ & 12 & $\begin{array}{c}1.68 \\
(1.01,2.79)\end{array}$ & 0.05 & 68 & $\begin{array}{l}\mathrm{RCT}[54,59] \\
(946)\end{array}$ & 12 & $\begin{array}{c}1.63 \\
(1.08,2.46)\end{array}$ & 0.02 & 52 & - & - & - & - & - \\
\hline Hypertension & $\begin{array}{l}\text { RCT }[54,59] \\
(946)\end{array}$ & 12 & $\begin{array}{c}0.98 \\
(0.73,1.32)\end{array}$ & 0.87 & 0 & $\begin{array}{l}\text { RCT }[54,59] \\
(946)\end{array}$ & 12 & $\begin{array}{c}0.97 \\
(0.80,1.18)\end{array}$ & 0.78 & 0 & - & - & - & - & - \\
\hline Lymphoceles & $\begin{array}{l}\text { RCT }[55,56] \\
(781)\end{array}$ & 36 & $\begin{array}{c}1.54 \\
(0.96,2.45)\end{array}$ & 0.07 & 14 & $\begin{array}{l}\mathrm{RCT}[55,56] \\
(780)\end{array}$ & 36 & $\begin{array}{c}2.08 \\
(1.00,4.32)\end{array}$ & 0.05 & 63 & - & - & - & - & - \\
\hline Withdraw & $\begin{array}{l}\mathrm{RCT}[55,56] \\
(781)\end{array}$ & 36 & $\begin{array}{c}1.23 \\
(1.07,1.43)\end{array}$ & 0.005 & 0 & $\begin{array}{l}\mathrm{RCT}[55,56] \\
(780)\end{array}$ & 36 & $\begin{array}{c}1.41 \\
(1.23,1.62)\end{array}$ & $<0.01$ & 0 & $\begin{array}{l}\text { RCT }[57,58] \\
(459)\end{array}$ & 12 & $\begin{array}{c}1.81 \\
(1.20,2.72)\end{array}$ & 0.004 & 0 \\
\hline
\end{tabular}

Abbreviations: (ld), Low dose; (hd), High dose; mo., months. ${ }^{a}$ Results reaching statistical significance are in bold font. ${ }^{\mathrm{b}}$ Relatives risk values of $<1$ favor treatment with TOR-I. ${ }^{\mathrm{c}} p$ : $p$-value for relative risk estimation; $\mathrm{I}^{2}$ : test for heterogeneity. 
Compared with CsA, treating kidney transplant patients with TAC resulted in a higher risk for diabetes, whereas those taking CsA had a greater risk of developing dyslipidemia. A retrospective study of risk factors for new-onset diabetes after transplantation (NODAT) found that higher tacrolimus concentrations were an independent predictor of NODAT [70]. In a meta-analysis comparing TAC vs. CsA as the primary immunosuppressant for kidney transplant recipients, TAC-treated patients were two to three times more likely to develop new diabetes mellitus that required insulin. However, the adverse events associated to CsA (constipation, hirsutism, and gingival hyperplasia) were different from those that we found, likely due to the time of use [16].

In regard to MMF vs. AZA, the majority of studies reported a larger number of adverse events for the groups treated with MMF at 12 months of follow-up. The meta-analyses of total infections, vomiting, diarrhea, and abdominal pains statistically favored treatment with AZA. The results of the present meta-analysis agree with the findings of a systematic review conducted in 2009 [71], which found that MMF-treated patients had a greater risk of diarrhea, whereas the risks of CMV infection, anemia, leukopenia and malignancy were not significant.

Our results showed that the use of SRL was associated with higher risk for anemia, dyslipidemia, lymphoceles and withdrawal compared with any CNI. There were no significant differences for infections, UTI, leukopenia, hypertension, or malignancies. These results agree with the findings of a multicenter study which used TAC in combination with different doses of SRL and showed that the incidence of dyslipidemia (hypercholesterolemia) was associated with higher doses of SRL [72]. Another study comparing TOR-I versus CNI found an increased risk of bone marrow suppression outcomes (leukopenia, thrombocytopenia, and anemia), lymphoceles and dyslipidemia for patients taking SRL [73]. Compared with CsA, SRL presented a higher risk for diabetes and reduced risk of CMV infection. Although regimens containing SRL have a higher risk of post-transplant diabetes than regimens without SRL [74], TAC has a higher risk for diabetes than CsA, thus the difference of risk between TAC and SRL may have no significance. Johnston et al. compared SRL with TAC and with CsA and found that patients treated with CsA had the lowest incidence of diabetes $(15.6 \%)$, followed by SRL (17.8\%) and then TAC (19\%) [74]. Sirolimus in combination with CNI may increase clinically significant adverse events, such as CNI-related nephrotoxicity and dyslipidemia. Other outcomes include hematologic side effects and a higher incidence of lymphoceles [75]. Furthermore, the use of SRL combined with TAC might increase the risk of post-transplant diabetes mellitus $[17,76]$.

As the majority of studies comparing TOR-I with CNI assess CNI minimization or elimination thru conversion from CNI to TOR-I, the number of studies with such comparison included in the present review was limited, once conversion of drugs was considered exclusion criteria.

Independent of the dose, EVL was associated with increased risk of dyslipidemia and withdrawal. MMF presented higher risk of CMV infection compared with both doses of EVL, but there was no difference in bone marrow suppression (leukopenia and anemia), hypertension, lymphoceles and infections. According to one study, SRL and MMF are associated with similar incidences of both leukopenia and thrombocytopenia [77]. This study reported similar incidences of leukopenia with the combination of MMF and SRL and MMF alone, and similar incidences of thrombocytopenia were observed between their combination and SRL alone, indicating no difference in the risk of these outcomes [77]. The definitions of diabetes and other diseases, such malignancies and dyslipidemia, vary a lot between studies, thus the interpretation of results regarding these diseases should consider 
the differences between definitions. Many of the clinical trials included were funded by pharmaceutical industries, limiting the interpretation of results, as these companies may benefit from reporting only favorable findings.

Current immunosuppressive protocols use combinations of immunosuppressive agents with different mechanisms of action to maximize efficacy and minimize the toxicity of each drug. The appearance of new immunosuppressive agents and tolerance protocols emerge shows potential as a means to deliver immunosuppression without long-term toxicity. In this regard, belatacept is a second-generation costimulation blocker that in phase 3 trials was to provide effective immunosuppression while avoiding the toxicities associated with calcineurin inhibitors [78].

Modifications are still being introduced in immunosuppressant protocols to take advantage of the drugs' beneficial actions and to reduce the adverse events. Although safety information alone is not enough to base decision making in health, together with reliable information about the long-term efficacy of immunosuppressants, the results of the present review might assist healthcare professionals and managers in choosing the best immunosuppressant regimen. We concluded that the data examined in this meta-analysis are similar to those describe by others authors. Adverse reactions were observed in all classes of immunosuppressive drugs; thus the choice of treatment must be made by the clinical staff based on specific patient characteristics.

\section{Acknowledgments}

The authors are grateful to the team of the Research Group in Pharmacoepidemiology (GPFE). This work received financial support from the Brazilian Ministry of Health and the Brazilian National Council for Scientific and Technological Development (CNPq).

\section{Conflicts of Interest}

The authors declare no conflict of interest.

\section{References}

1. Levey, A.S.; Coresh, J.; Balk, E.; Kausz, A.T.; Levin, A.; Steffes, M.W.; Hogg, R.J.; Perrone, R.D.; Lau, J.; Eknoyean, G. National Kidney Foundation practice guidelines for chronic kidney disease: Evaluation, classification, and stratification. Ann. Intern. Med. 2003, 139, 137-147.

2. Coresh, J.; Selvin, E.; Stevens, L.A.; Manzi, J.; Kusek, J.W.; Eggers, P.; van Lente, F.; Levey, A.S. Prevalence of chronic kidney disease in the United States. JAMA 2007, 298, 2038-2046.

3. Zhang, Q.L.; Rothenbacher, D. Prevalence of chronic disease in population-based studies: Systematic review. BMC Public Health 2008, 8, 117.

4. Nagata, M.; Ninomiya, T.; Doi, Y.; Yonemoto, K.; Kubo, M.; Hata, J.; Tsuruya, K.; Iida, M.; Kiyohara, Y. Trends in the prevalence of chronic kidney disease and its risk factors in a general Japanese population: The Hisayama study. Nephrol. Dial. Transplant. 2010, 25, 2557-2564.

5. Van Pottelbergh, G.; Bartholomeeusen, S.; Buntinx, F.; Degryse, J. The prevalence of chronic kidney disease in a Flemish primary care morbidity register. Age Ageing 2011, 41, 231-233. 
6. Muntner, P.; Newsome, B.; Kramer, H.; Peralta, C.A.; Kim, Y.; Jacobs, D.R., Jr.; Kiefe, C.I.; Lewis, C.E. Racial Differences in the Incidence of Chronic Kidney Disease. Clin J. Am. Soc. Nephrol. 2011, 7, 101-107.

7. McCullough, K.; Sharma, P.; Ali, T.; Khan, I.; Smith, W.C.S.; MacLeod, A.; Black, C. Measuring the population burden of chronic kidney disease: A systematic literature review of the estimated prevalence of impaired kidney function. Nephrol. Dial. Transplant. 2012, 27, 1812-1821.

8. Levey, A.S.; Atkins, R.; Coresh, J.; Cohen, E.P.; Collins, A.J.; Eckardt, K.U.; Nahas, M.E.; Jaber, B.L.; Jadoul, M.; Levin, A.; et al. Chronic kidney disease as a global public health problem: Approaches and initiatives-A position statement from Kidney Disease Improving Global Outcomes. Kidney Int. 2007, 72, 247-259.

9. Cusumano, A.M.; Gonza'lez Bedat, M.C. Chronic Kidney Disease in Latin America: Time to improve screening and detection. Clin. J. Am. Soc. Nephrol. 2008, 3, 594-600.

10. Port, F.K.; Wolfe, R.A.; Mauger, E.A.; Berling, D.P.; Jiang, K. Comparison of survival probabilities for dialysis patients vs. cadaveric renal transplantation recipients. JAMA 1993, 270, 1339-1343.

11. Evans, R.W.; Manninen, D.L.; Garrison, L.P.; Hart, L.G; Blagg, C.R.; Gutman, R.A.; Hull, A.R.; Lowrie, E.G. The quality of life of patients with end-stage renal disease. N. Engl. J. Med. 1985, 312, 553-559.

12. Eggers, P.W. Effect of transplantation on the Medicare End-Stage Renal Disease Program. N. Engl. J. Med. 1988, 318, 223-229.

13. National Kidney and Urologic Diseases Information Clearinghouse. Kidney and Urologic Diseases Statistics for the United States. Available online: http://kidney.niddk.nih.gov/kudiseases/ pubs/kustats/ (accessed on 1 May 2012).

14. Kidney Disease: Improving Global Outcomes (KDIGO). Clinical practice guideline for the care of kidney transplant recipients. Am. J. Transplant. 2009, 9, S1-S155.

15. Sprangers, B.; Kuypers, D.R.; Vanrenterghem, Y. Immunosuppression: Does on regimen fit all? Transplantation 2011, 92, 251-261

16. Liberati, A.; Altman, D.G.; Tetzlaff, J.; Mulrow, C.; Gotzsche, P.C.; Loannidis, J.P.A.; Clarke, M.; Devereaux, J.K.; Moher, D. The PRISMA statement for reporting systematic reviews and meta-analyses of studies that evaluate health care interventions: Explanation and elaboration. PLoS Med. 2009, 6, 7:1-7:28.

17. Woodroffe, R.; Yao, G.L.; Meads, C.; Bayliss, S.; Ready, A.; Raftery, J.; Taylor, R.S. Clinical and cost-effectiveness of newer immunosuppressive regimens in renal transplantation: A systematic review and modelling study. Health Technol. Assess. 2005, 9, 21:1-21:179.

18. Webster, A.C.; Woodroffe, R.C.; Taylor, R.S.; Chapman, J.R.; Craig, J.C. Tacrolimo versus ciclosporin as primary immunosuppression for kidney transplant recipients: Meta-analysis and meta-regression of randomised trial data. BMJ 2005, 331, 810:1-810:11.

19. Cochrane Handbook for Systematic Reviews of Interventions, Version 5.1.0. Available online: www.cochrane-handbook.org/ (accessed on 24 June 2013).

20. Newcastle-Ottawa Scale (NOS) for Assessing the Quality of Nonrandomized Studies in Meta-analyses. Available online: http://www.ohri.ca/programs/clinical_epidemiology/oxford.htm/ (accessed on 6 August 2010). 
21. Higgins, J.P.T.; Thompson, S.G.; Deeks, J.J.; Altman, D.G. Measuring inconsistency in meta-analyses. BMJ 2003, 327, 557-560.

22. Scantleburry, V.; Shapiro, R.; Fung, J.; Tzakis, A.; McCauley, J.; Jordan, M.; Jensen, C.; Hakala, T.; Simmons, R.; Starzi, T.E. New onset of diabetes in FK 506 vs. cyclosporine-treated kidney transplant recipients. Transplant. Proc. 1991, 23, 3169-3170.

23. Mayer, A.D.; Dmitrewski, J.; Squifflet, J.P.; Bese, T.; Grabensee, B.; Klein, B.; Eigler, F.W.; Heemann, U.; Pichlmayr, R.; Behrend, M. Multicenter randomized trial comparing tacrolimus (FK506) and cyclosporine in the prevention of renal allograft rejection: A report of the European Tacrolimus Multicenter Renal Study Group. Transplantation 1997, 64, 436-443.

24. Yang, H.C.; Holman, M.J.; Langhoff, E.; Ulsh, P.J.; Dellock, C.A.; Gupta, M.; Ahsan, N. Tacrolimus/"low-dose" mycophenolate mofetil versus microemulsion cyclosporine/"low-dose" mycophenolate mofetil after kidney transplantation: 1-year follow-up of a prospective, randomized, clinical trial. Transplant. Proc. 1999, 31, 1121-1124.

25. Wang, X.H.; Tang, X. D.; Xu, D. Tacrolimus vs. Cya Neoral in Combination with MMF and steroid after cadaveric renal transplantation. Transplant. Proc. 2000, 32, 1702-1703.

26. Nichelle, L.; Canet, S.; Garrigue, V.; Chong, G.; Mourad, G. Arterial hypertension in renal transplant recipients treated with tacrolimus or cyclosporine-Neoral. Transplant. Proc. 2002, 34, 2824-2825.

27. Campos, H.H.; Abbud, M.F. One-year follow-up of a brazilian randomized multicenter study comparing tacrolimus versus cyclosporine in kidney transplantation. Transplant. Proc. 2002, 34, 1656-1658.

28. Murphy, G.J.; Waller, J.R.; Sandford, R.S.; Furness, P.N.; Nicholson, M.L. Randomized clinical trial of the effect of microemulsion cyclosporine and tacrolimus on renal allograft fibrosis. $\mathrm{Br}$. $J$. Surg. 2003, 90, 680-686.

29. Hardinger, K.L.; Bohl, D.L.; Schnitzler, M.A.; Lockwood, M.; Storch, G.A.; Brennan, D.C. A randomized, prospective, pharcoeconomic trial of tacrolimus versus cyclosporine in combination with thymoglobulin in renal transplant recipients. Transplantation 2005, 80, 41-46.

30. Silva, H.T., Jr.; Yang, H.C.; Abouljoud, M.; Kuo, P.C.; Wisemandle, K.; Bhattacharya, P.; Dhadda, S.; Holman, J.; Fitzsimmoms, W.; First, M.R. One-year results with extended-release tacrolimus/MMF, tacrolimus/MMF and cyclosporine/MMF in de novo kidney transplant recipients. Am. J. Transplant. 2007, 7, 595-608.

31. Cheung, C.Y.; Chan, H.W.; Liu, Y.L.; Chau, K.F.; Li, C.S. Long-term graft function with tacrolimus and cyclosporine in renal transplantation: Paired kidney analysis. Nephrology 2009, $14,758-763$.

32. Vicenti, F.; Laskow, D.A.; Neylan, J.F.; Mendez, R.; Matas, A.J. One-year follow-up of an open-label trial of Fk506 for primary kidney transplantation: A report of the U.S. multicenter fk506 kidney transplant group. Transplantation 1996, 61, 1576-1581.

33. Moreso, F.; Serón, D.; Morales, J.M.; Cruzado, J.M.; Gil-Vernet, S.; Pérez, J.L.; Fulladosa, X.; Andrés, A.; Grinyó, J.M. Incidence of leucopenia and citomegalovirus disease in kidney transplants treated with mycophenolate mofetil combined with low cyclosporine and steroid doses. Clin. Transplant. 1998, 12, 198-205. 
34. Raofi, V.; Holman, D.M.; Coady, N.; Vazquez, E.; Dunn, T.B.; Bartholomew, A.M.; Pollak, R.; Benedetti, E. A prospective randomized trial comparing the efficacy of tacrolimus versus cyclosporine in black recipients of primary cadaveric renal transplants. Am. J. Surg. 1999, 177, 299-302.

35. Sandrini, S.; Maiorca, R.; Scolari, F.; Cancarini, G.; Setti, G.; Gaggia, P.; Cristinelli, L.; Zubani, R.; Bonardelli, S.; Maffeis, R.; et al. A prospective randomized trial on azathioprine addition to cyclosporine versus cyclosporine monotherapy at steroid withdrawal, 6 months after renal transplantation. Transplantation 2000, 69, 1861-1867.

36. Segoloni, G.; Bonomini, V.; Maresca, M.C.; Arisi, L.; Gonzales-Molina, M.; Tarantino, A.; del Castillo, D.; Ortuño, J.; Carmellini, M.; Capdevila, L.; et al. Tacrolimus is highly effective in bith dual and triple therapy regimens following renal transplantation. Transpl. Int. 2000, 13, S336-S340.

37. Pascual, J.; Segoloni, G.; Gonzales-Molina, G.; del Castillo, D.; Capdevila, L.; Arias, M.; Garcia, J.; Ortuño, J. Comparison between a two-drug regimen with tacrolimus and steroids and a triple one with azathioprine in kidney tansplantation: Results of a European Trial with 3-year follow up. Transplant. Proc. 2003, 35, 1701-1703.

38. Chang, R.W.S.; Snowden, S.; Palmer, A.; Kwan, J.T.C.; Nocholson, M.; Kashi, S.H.; Fernando, O.N.; Perner, F.; Neild, G.H. European randomized trial of dual versus triple tacrolimus-based regimens for controlof acute rejection in renal allograft recipients. Transpl. Int. 2001, 14, 384-390.

39. Squifflet, J.P.; Bäckman, L.; Claesson, K.; Dieth, K.H.; Ekberg, H.; Dorsythe, J.L.R.; Kunsendorf, U.; Heemann, U.; Land, W.; Morales, J.M.; et al. Dose Optimization of a Micophenolate Mofetil when administered with a low dose of tacrolimus in cadaveric renal transplant recipients. Transplantation 2001, 72, 63-69.

40. Hall, B.M.; Tiller, D.J.; Hardie, I.; Mahony, J.; Mathew, T.; Thatcher, G.; Miach, P.; Thomson, N.; Sheil, A.G. Comparison of three immunosuppressive regimens in cadaver renal transplantation: Long-term cyclosporine, short-term cyclosposrine followed by azathioprine and prednisolone, and azathioprine and prednisolone without cyclosporine. N. Engl. J. Med. 1988, 318, 1499-1507.

41. Schnuelle, P.; van der Heide, J.H.; Tegzess, A.; Verburgh, C.A.; Paul, L.C.; van der Woude, F.J.; de Fijter, J.W. Open randomized trial comparing early withdrawal of either cyclosporine or micophenolate mofetil in stable renal transplant recipients initially treated with a triple drug regimen. J. Am. Soc. Nephrol. 2002, 13, 536-543.

42. Hamdy, A.F.; Bakr, M.A.; Ghoneim, M.A. Long-term efficacy and safety of a calcineurin inhibitor-free regimen in live-donor renal transplant recipients. J. Am. Soc. Nephrol. 2008, 19, 1225-1232.

43. Groth, C.G.; Bäckman, L.; Morales, J.M.; Kreis, H.; Lang, P.; Touraine, J.L.; Claesson, K.; Campistol, J.M.; Durand, D.; Wramner, L.; et al. Sirolimus (rapamycin)-based therapy in human renal transplantation: Similar efficacy and different toxicity compared with cyclosporine. Transplantation 1999, 67, 1036-1042.

44. Büchler, M.; Caillard, S.; Barbier, S.; Thervet, E.; Toupance, O.; Mazouz, H.; de Ligny, D.H.; Le Meur, Y.; Thierry, A.; Villemain, F.; et al. Sirolimus versus cyclosporine in kidney recipients receiving thymoglobulin, mycophenolate mofetil and a 6-month course of steroids. Am. J. Transplant. 2007, 7, 2522-2531. 
45. Lebranchu, Y.; Snanoudj, R.; Toupance, O.; Weestel, P.F.; Hurault de Ligny, B.; Buchler, M.; Rerolle, J.P.; Thierry, A.; Moulin, B.; Subra, J.F.; et al. Five-year results of a randomized trial comparing de novo sirolimus and cyclosporine in renal transplantation: The SPIESSER study. Am. J. Transplant. 2012, 12, 1801-1810.

46. Guba, M.; Pratschke, J.; Hugo, C.; Krämer, B.K.; Nohr-Westphal, C.; Brockman, J.; Andrassy, J.; Reinke, P.; Pressmar, K.; Hakenberg, O.; et al. Renal function, efficacy, and safety of sirolimus ans mycophenolate mofetil after short-term calcineurin inhibitor-based quadruple therapy in de novo renal transplant patients: One-year analysis of a randomized multicenter trial. Tansplantation 2010, 90, 175-183.

47. Glotz, D.; Charpentier, B.; Abramovicz, D.; Lang, P.; Rostaing, L.; Rifle, G.; Vanrenterghem, Y.; Bethous, F.; Bourbigot, B.; Delahousse, M.; et al. Thymoglobulin induction and sirolimus versus tacrolimus in kidney transplant recipients receiving mycophenolate mofetil and steroids. Transplantation 2010, 89, 1511-1517.

48. Hernández, D.; Miquel, R.; Porrini, E.; Fernández, A.; González-Posada, J.; Hortal, L.; Checa, M.D.; Rodríguez, A.; García, J.J.; Rufino, M.; et al. Randomized controlled study comparing reduced calcineurin inhibitors exposure versus standard cyclosporine-based immunosuppression. Transplantation 2007, 84, 706-714.

49. Keown, P.; Häyry, P.; Mathew, T.; Morris, P.; Stiller, C.; Barker, C.; Carr, L. A blinded, randomized clinical trial of mycophenolate mofetil for the prevention of acute rejection in cadaveric renal transplantation. The tricontinental mycophenolate mofetil renal transplantation study group. Transplantation 1996, 61, 1029-1037.

50. Pescovitz, M.; Conti, D.; Greenstein, S.M.; Inokuchi, S.; Rosenthal, J.; Cohen, D.; Stiller, C.; Pirsch, J.; Tomlanovich, S.; Cho, S.; et al. Mycophenolate Mofetil for The Treatment of a first acute renal allograft rejection. The tricontinental mycophenolate mofetil renal transplantation study group. Transplantation 1998, 65, 235-241.

51. Pescovitz, M.; Conti, D.; Greenstein, S.M.; Inokuchi, S.; Rosenthal, J.; Cohen, D.; Stiller, C.; Pirsch, J.; Tomlanovich, S.; Cho, S.; et al. Mycophenolate mofetil for the treatment of a first acute renal allograft rejection: Three-year follow-up. The mycophenolate mofetil acute renal rejection study group. Transplantation 2001, 71, 1091-1097.

52. Folkmane, I.; Bicans, J.; Chapenko, S.; Murovska, M.; Rosentals, R. Results of renal transplantation with different immunosuppressive regimens. Transplant. Proc. 2002, 34, 558-559.

53. Sadek, S.; Medina, J.; Arias, M.; Sennesael, J.; Squifflet, J.P.; Vogt, B. Short-term combination of mycophenolate mofetil with cyclosporine as a therapeutic option for renal transplant recipients. Transplantation 2002, 74, 511-517.

54. Vítko, S.; Margreiter, R.; Weimar, W.; Dantal, J.; Viljoen, H.G.; Li, Y.; Jappe, A.; Cretin, N. Everolimus (Certican) 12-month safety efficacy versus mycophenolate mofetil in de novo renal transplant recipients. Transplantation 2004, 78, 1532-1540.

55. V'́tko, S.; Margreiter, R.; Weimar, W.; Dantal, J.; Kuypers, D.; Winkler, M.; Oyen, O.; Viljoen, H.G.; Filiptsev, P.; Sadek, S.; et al. Three-year efficacy and safety results form a study of everolimus versus mycophenolate mofetil in de novo renal transplant patients. Am. J. Transplant. 2005, 5, 2521-2530. 
56. Lorber, M.I.; Mulgaonkar, S.; Butt, K.M.H.; Elkhammas, E.; Mendez, R.; Rajagopalan, P.R.; Kahan, B.; Solinger, H.; Li, Y.; Cretin, N.; et al. Everolimus versus mycophenolate mofetil in the prevention of rejection in de novo renal transplant recipients: A 3-year randomized, multicenter phase III study. Transplantation 2005, 80, 244-252.

57. Mendez, R.; Gonwa, T.; Yang, H.C.; Weinstein, S.; Jensik, S.; Steinberg, S. A prospective, randomized trial of tacrolimus in combination with sirolimus or mycophenolate mofetil in kidney transplantation: Results at 1 year. Transplantation 2005, 80, 303-309.

58. Sampaio, E.L.; Pinheiro-Machado, P.G.; Garcia, R.; Felipe, C.R.; Park, S.I.; Casarini, D.E.; Moreira, S.; Franco, M.F.; Tedesco-Silva, H., Jr.; Medina-Pestana, J.O. Mycophenolate mofetil vs. sirolimus in kidney transplant recipients receiving tacrolimus-based immunosuppressive regimen. Clin. Transplant. 2008, 22, 141-149.

59. Tedesco-Silva, H., Jr.; Cibrik, D.; Johnston, T.; Lackova, E.; Mange, K.; Pamos, C.; Walker, R.; Wang, Z.; Zibari, G.; Kim, Y.S. Everolimus plus reduced-exposure CsA versus mycophenolic acid plus standard exposure CsA in renal-transplant patients. Am. J. Transplant. 2010, 10, 1401-1413.

60. Cibrik, D.; Silva, H.T., Jr; Vathsala, A.; Lackova, E.; Cornu-Artis, C.; Walker, RG.; Wang, Z.; Zibari, G.B.; Shihab, F.; Kim, Y.S. Randomized trial of everolimus-facilitated calcineurin inhibitor minimization over 24 months in renal transplantation. Transplantation 2013, 95, 933-942.

61. Ekberg, H.; Tedesco-Silva, H.; Demirbas, A.; Vitko, S.; Nashan, B.; Gürkan, A.; Margreiter, R.; Hugo, C.; Grinyó, J.M.; Frei, U.; et al. Reduced exposure to calcineurin inhibitors in renal transplantation. N. Engl. J. Med. 2007, 357, 2562-2575.

62. Ekberg, H.; Bernasconi, C.; Tedesco-Silva, H.; Vitko, S.; Hugo, C.; Demirbas, A.; Acevedo, R.R.; Grinyó, J.; Frei, U.; Vamrenterghem, Y.; et al. Calcineurin inhibitor minimization in the Symphony Study: Observational results 3 years after transplantation. Am. J. Transplant. 2009, 9, 1876-1885.

63. Kumar, M.S.A.; Moritz, M.J.; Saaed, M.I.; Heifets, M.; Sustento-Reodica, N.; Fyfe, B.; Kumar, A. Avoidance of chronic steroid therapy in african american kidney transplant recipients monitored by surveilance biopsy: 1-year results. Am. J. Transplant. 2005, 5, 1976-1985.

64. Tedesco-Silva, H.; Garcia, V.D.; Contieri, F.L.C.; Carvalho, D.B.M.; Noronha, I.L.; Gonçalves, R.T.; de Paula, F.J.; Abbud-Filho, M.; Manfro, R.C.; David-Neto, R. Comparison of the safety and efficacy of cyclosporine minimization versus cyclosporine elimination in de novo renal allograft patients receiving sirolimus. Transplant. Proc .2010, 42, 1659-1666.

65. Flechner, S.M.; Glyda, M.; Cockfield, S.; Grinyó, J.; Legendre, C.; Russ, G.; Steinberg, S.; Wissing, K.M.; Tai, S.S. The ORION study: Comparison of two sirolimus-based regimens versus tacrolimus and mycophenolate mofetil in renal allograft recipients. Am. J. Transplant. 2011, 11, $1633-1644$.

66. Jurewicz, W.A. Tacrolimus versus ciclosporin immunosuppression: Long-term outcome in renal transplantation. Nephrol. Dial. Transplant. 2003, 18, i7-i11.

67. Fukuhara, N.; Ono, Y.; Hattori, R.; Nishiyama, N.; Yamada, S.; Kamihira, O.; Kinukawa, T.; Ohshima, S. The long-term outcome of tacrolimus in cadaveric kidney transplantation form non-heart beating donors. Clin. Transplant. 2005, 19, 153-157. 
68. Silva, L.A.; Felipe, C.R.; Park, S.I.; Pinheiro-Machado, P.; Garcia, R.; Franco, M.; Moreira, S.R.; Tedesco-Silva, H., Jr.; Medina-Pestana, J. Impact of initial exposure to calcineurin inhibitors on kidney graft function of patients at high risk to develop delayed graft function. Braz. J. Med. Biol. Res. 2006, 39, 43-52.

69. Gheith, O.A.; Bakr, M.A.; Fouda, M.A.; Shokeir, A.A.; Bayoumy, A.; Sobh, M.; Ghoneim, M. Steroid and azathioprine versus steroid, cyclosporine, and azathioprine therapies in primary haplo-identical living donor kidney transplantation: Twenty-year experience. Iran J. Kidney Dis. 2008, 2, 34-39.

70. Cotovio, P.; Neves, M.; Rodrigues, L.; Alves, R.; Bastos, M.; Baptista, C.; Macário, F.; Mota, A. New-onset diabetes after transplantation: Assessment of risk factors and clinical outcomes. Transplant. Proc. 2013, 45, 1079-1083.

71. Knight, S.R.; Russell, N.K.; Barcena, L.; Morris, P.J. Mycophenolate mofetil decreases acute rejection and may improve graft survival in renal transplant recipients when compared with azathioprine: A systematic review. Transplantation 2009, 87, 785-794.

72. Van Hooff, J.P.; Squifflet, J.P. A prospective randomized multicenter study of tacrolimus in combination with sirolimus in renal transplant recipients. Transplantation 2003, 75, 1934-1939.

73. Webster, A.C.; Lee, V.W.; Chapman, J.R.; Craig, J.C. Target of rapamycin inhibitors (sirolimus and everolimus) for primary immunosuppression of kidney transplant recipients: A systematic review and meta-analysis of randomized trials. Transplantation 2006, 81, 1234-1248.

74. Johnston, O.; Rose, C.L.; Webster, A.C.; Gill, J.S. Sirolimus is associated with new-onset diabetes in kidney transplant recipients. J. Am. Soc. Nephrol. 2008, 19, 1411-1418.

75. Danovitch, G.M. Immunosuppressive Medications and Protocols for Kidney Transplantation. In Handbook of Kidney Transplantation, 4th ed.; Danovitch, G.M., Eds.; Lippincott Williams \& Wilkins: Philadelphia, PA, USA, 2005, pp. 72-134.

76. Ribes, D.; Kamar, N. Combined use of tacrolimus and sirolimus in de novo renal transplant patients: Current data. Transplant. Proc. 2005, 37, 2813-2816

77. Gralla, J.; Wiseman, A.C. Tacrolimus/sirolimus versus tacrolimus/mycophenolate in kidney transplantation: Improved 3-year graft and patient survival in recent era. Transplantation 2009, 87, 1712-1719.

78. Yabu, J.M.; Vincenti, F. Kidney Transplantation: The ideal immunosuppression regimen. Adv. Chronic Kidney Dis. 2009, 16, 226-233.

(C) 2013 by the authors; licensee MDPI, Basel, Switzerland. This article is an open access article distributed under the terms and conditions of the Creative Commons Attribution license (http://creativecommons.org/licenses/by/3.0/). 